\title{
A filtration problem through a heterogeneous porous medium
}

\author{
S. CHALLAL ${ }^{\dagger}$ AND A. LYAGHFouri ${ }^{\ddagger}$ \\ King Fahd University of Petroleum and Minerals, \\ P.O. Box 728, Dhahran 31261, Saudi Arabia
}

[Received 25 May 2002 and in revised form 9 November 2003]

\begin{abstract}
The flow of a fluid through a heterogeneous porous medium is studied, assuming it is governed by a nonlinear Darcy law and Dirichlet boundary conditions. Under a general condition on the permeability we prove that the free boundary is locally a continuous curve in some local coordinates. We also prove the uniqueness of the reservoirs-connected solution.
\end{abstract}

\section{Introduction}

The dam problem has attracted the attention of many researchers over the last thirty years. However there still exist a number of unsolved questions related to this challenging problem, including the regularity of the free boundary and the uniqueness of the solution for flows in general heterogeneous porous media.

To begin with we would like to say a few words about the history of the problem; for brevity, we restrict ourselves to the steady state case with Dirichlet boundary conditions on the bottoms of the reservoirs.

First Baiocchi solved in [6] (see also [7] and [31]) the case of rectangular dams by using variational inequalities. For dams with general geometry a new approach was introduced by $\mathrm{H}$. W. Alt in [3] for the heterogeneous case and by H. Brezis, D. Kinderlehrer, and G. Stampacchia in [12] for the homogeneous case. The two formulations are equivalent to

$$
\left(\mathrm{P}_{1}\right)\left\{\begin{array}{l}
\text { Find }(p, \chi) \in H^{1}(\Omega) \times L^{\infty}(\Omega) \text { such that: } \\
\text { (i) } p \geqslant 0, \quad 0 \leqslant \chi \leqslant 1, \quad p(1-\chi)=0 \quad \text { a.e. in } \Omega, \\
\text { (ii) } p=\varphi \quad \text { on } S_{2} \cup S_{3}, \\
\text { (iii) } \int_{\Omega} a(X)(\nabla p+\chi e) \cdot \nabla \xi \mathrm{d} X \leqslant 0, \quad e=(0,1), \\
\quad \text { for all } \xi \in H^{1}(\Omega) \text { with } \xi=0 \text { on } S_{3} \text { and } \xi \geqslant 0 \text { on } S_{2},
\end{array}\right.
$$

where $p$ is the fluid pressure, $\chi$ a function characterizing the wet part of the dam, $a(X)=\left(a_{i j}(X)\right)$ is the permeability matrix of the medium and $X=(x, y)$. The existence of a solution $(p, \chi)$ was proved. Concerning the regularity of the free boundary, H. W. Alt proved in [4] that in the homogeneous case it is an analytic curve $y=\Phi(x)$. Uniqueness of the so-called $S_{3}$-connected solution was proved by J. Carrillo and M. Chipot in [14] and also by H.W. Alt and G. Gilardi in [5].

In [15], J. Carrillo and A. Lyaghfouri considered this problem, assuming the flow governed by the following nonlinear Darcy law (see [22]):

$$
|v|^{m-1} v=-\nabla(p+y), \quad m>0 .
$$

\footnotetext{
†Email: challal.samia@caramail.com
}

Email: lyaghfo@kfupm.edu.sa 
They formulated the problem in terms of the hydrostatic head $u=p+y$ and were led to an extension of $\left(\mathrm{P}_{1}\right)$ corresponding to the general problem $(\mathrm{P})$ given in the next section, with $\mathcal{A}(X, \xi)=|\xi|^{q-2} \xi$ and $q=1+1 / m$. Despite the nonlinearity, the authors showed that this problem is well posed and proved the existence of a solution, the continuity of the free boundary $y=\Phi(x)$ and the uniqueness of the $S_{3}$-connected solution in the case $n=2$. For $n \geqslant \max (2, q)$, they proved existence and uniqueness of a minimal solution.

The case of a general heterogeneous dam of general geometry was formulated first in [3] by H. W. Alt who proved the existence of a solution and local Lipschitz continuity of the pressure. Moreover he gave a counterexample showing that $\chi$ may not be the characteristic function of the wet set $[p>0]$. He also proved that

$$
\operatorname{div}\left(a_{12}, a_{22}\right) \geqslant 0 \text { in } \mathcal{D}^{\prime}(\Omega) \Rightarrow \nabla \chi \cdot a(X)(e) \leqslant 0 \text { in } \mathcal{D}^{\prime}(\Omega) .
$$

In [21] and [32], the authors showed that if $a(X)=k(x, y) I_{2}$ with $\partial k / \partial y \geqslant 0$ in $\mathcal{D}^{\prime}(\Omega)$, then the free boundary is a continuous curve $y=\Phi(x)$ and the $S_{3}$-connected solution is unique. These results were generalized by the second author in [27] to the case where

$$
a(X)=\left(\begin{array}{lc}
a_{11}(X) & 0 \\
a_{21}(X) & a_{22}(X)
\end{array}\right) \text { and } \frac{\partial a_{22}}{\partial y} \geqslant 0 \text { in } \mathcal{D}^{\prime}(\Omega)
$$

From the description of the heterogeneous case, the following natural question arises: Can we always describe the free boundary globally or at least locally as the graph of a continuous function, that is, is it necessarily of the form $y=\Phi(x)$ or $x=\Psi(y)$ ?

It is our purpose in this paper to address this issue in the more general case where the flow is governed by the nonlinear law

$$
v=-\mathcal{A}(X, \nabla(p+y)) .
$$

Then by using a similar formulation to [15], i.e. $u=p+y$ and $g=1-\chi$, and by assuming that

$$
\operatorname{div}(\mathcal{A}(X, e)) \geqslant 0 \text { in } \mathcal{D}^{\prime}(\Omega) \text { and } \mathcal{A}(X, e) \in C^{1}(\bar{\Omega}),
$$

we give a positive answer to the above question.

The main new idea is the following: we remark that under the above assumption, the function $g$ is nondecreasing along the orbits of the ordinary differential equation

$$
X^{\prime}(t)=\mathcal{A}(X(t), e)
$$

which generalizes the fact that $\chi$ is nonincreasing with respect to the second variable $y$ when $a_{12}=$ 0 and $a_{22}$ is nondecreasing with respect to $y$ (see [27]). It follows that if the pressure is positive at some point $X_{0}=X\left(t_{0}\right)$ of the porous medium, where $X(\cdot)$ is the orbit containing $X_{0}$, then

$$
p(X(t))>0 \quad \forall t \leqslant t_{0} .
$$

This important property is then exploited to prove that the free boundary is represented locally by continuous graphs. This is done essentially by introducing two $C^{1}$-diffeomorphisms related to the above ordinary differential equation. As a consequence we deduce that $g$ is the characteristic function of the dry part $[p=0]$. This helps to show the uniqueness of the $S_{3}$-connected solution which we prefer here to call the reservoirs-connected solution. 
We would like to point out that in all previous studies, the dams considered are enclosed between two curves $y=s_{-}(x)$ and $y=s_{+}(x)$ which represent respectively the bottom and top of the dam. This implicitly assumes that the dam is vertically convex. In this study we do not assume this constraint and allow a wide variety of geometrical forms for our dam. We recall that for the existence of a solution it is only required that $\Omega$ is locally Lipschitz. However for the study of the free boundary we will assume that $\Omega$ is locally of class $C^{1}$. Finally we have chosen to introduce various hypotheses gradually into the text as the need arises.

The paper is organized as follows: in Section 1, we give the weak formulation of the problem and some of its properties. In Section 2, we prove a monotonicity property for the function $g$. In Section 3, we define a family $\left(\Phi_{h}\right)_{h}$ of functions representing locally the free boundary and prove they are lower semicontinuous. In Section 4, we prove some useful lemmas. In Section 5, we prove the continuity of the functions $\Phi_{h}$. Finally in Section 6, we prove the uniqueness of the reservoirsconnected solution.

\section{Formulation of the problem}

A porous medium that we denote by $\Omega$ is supplied by several reservoirs of a fluid which infiltrates through $\Omega$. We assume that $\Omega$ is a bounded locally Lipschitz domain of $\mathbb{R}^{2}$ with boundary $\partial \Omega=$ $S_{1} \cup S_{2} \cup S_{3}$, where $S_{1}$ is the impervious part, $S_{2}$ is the part in contact with air and $S_{3}=\bigcup_{i=1}^{N} S_{3, i}$ with $S_{3, i}(i=1, \ldots, N)$ the part in contact with the bottom of the $i^{\text {th }}$ reservoir. We assume that the flow in $\Omega$ has reached a steady state and we look for the fluid pressure $p$ and the saturated region $S$ of the porous medium. The boundary $\partial S$ of $S$ is divided into four parts (see Figure 1):

- $\Gamma_{1} \subset S_{1}$ : the impervious part,

- $\Gamma_{2} \subset \Omega$ : the free boundary,

- $\Gamma_{3} \subset S_{3}$ : the part covered by fluid,

- $\Gamma_{4} \subset S_{2}$ : the part where the fluid flows outside $\Omega$.

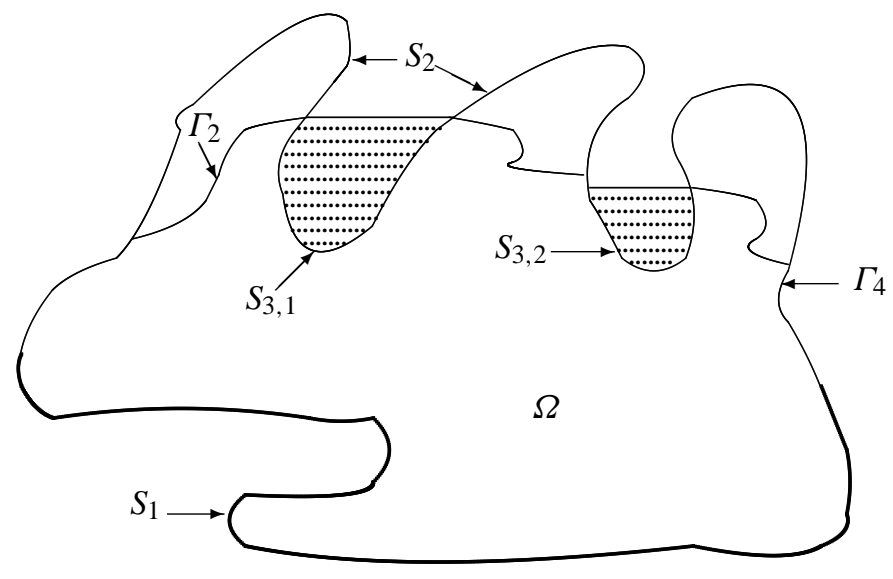

FIG. 1

The flow is governed by the following nonlinear Darcy law:

$$
v=-\mathcal{A}(X, \nabla(p+y))=-\mathcal{A}(X, \nabla u),
$$


where $v$ is the fluid velocity, $u=p+y$ is the hydrostatic head and $\mathcal{A}: \Omega \times \mathbb{R}^{2} \rightarrow \mathbb{R}^{2}$ is a mapping that satisfies the following assumptions for some constants $q>1$ and $0<\lambda \leqslant M<\infty$ :

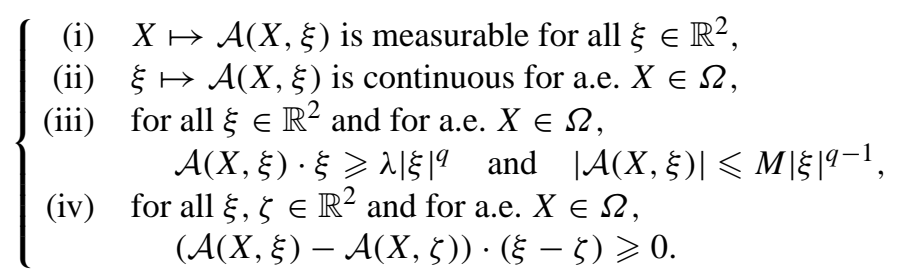

Moreover we have the following boundary conditions:

$$
\begin{cases}p=0 \text { on } S_{2}, \quad p=\varphi \text { on } S_{3}, & v \cdot v=0 \text { on } \Gamma_{1} \\ p=0 \text { and } v \cdot v=0 \text { on } \Gamma_{2}, & v \cdot v \geqslant 0 \text { on } \Gamma_{4}\end{cases}
$$

where $\varphi$ is a nonnegative Lipschitz continuous function which represents the fluid pressure at the bottoms of the reservoirs. For convenience we assume that $S_{3}$ is open relatively to $\partial \Omega$.

Assuming the flow to be incompressible and taking into account (1.1) and (1.3), we are led (see [15]) to the following problem:

$$
\text { (P) }\left\{\begin{aligned}
\text { Find }(u, g) \in W^{1, q}(\Omega) \times L^{\infty}(\Omega) \text { such that: } \\
\text { (i) } u \geqslant y, \quad 0 \leqslant g \leqslant 1, \quad g(u-y)=0 \quad \text { a.e. in } \Omega \\
\text { (ii) } u=\varphi+y \text { on } S_{2} \cup S_{3}, \\
\text { (iii) } \int_{\Omega}(\mathcal{A}(X, \nabla u)-g \mathcal{A}(X, e)) \cdot \nabla \xi d X \leqslant 0 \\
\quad \text { for all } \xi \in W^{1, q}(\Omega) \text { with } \xi=0 \text { on } S_{3} \text { and } \xi \geqslant 0 \text { on } S_{2} .
\end{aligned}\right.
$$

For the existence of a solution of (P) under the assumptions (1.2), we refer the reader to [29] where an existence result is given for more general boundary conditions. The reader can also adapt the proof in [15] obtained for the case $\mathcal{A}(X, \xi)=|\xi|^{q-2} \xi$.

Arguing as in [17] or [27], we obtain

Proposition 1.1 For each solution $(u, g)$ of $(\mathrm{P})$, we have

$$
\operatorname{div}(\mathcal{A}(X, \nabla u)-g \mathcal{A}(X, e))=0 \quad \text { in } \mathcal{D}^{\prime}(\Omega) .
$$

Moreover if $\operatorname{div}(\mathcal{A}(X, e)) \geqslant 0$ in $\mathcal{D}^{\prime}(\Omega)$, we obtain

$$
\operatorname{div}(\mathcal{A}(X, \nabla u))=\operatorname{div}(g \mathcal{A}(X, e)) \geqslant 0 \quad \text { in } \mathcal{D}^{\prime}(\Omega) .
$$

\section{A monotonicity property of $g$}

From now on, we shall assume that

$$
\begin{gathered}
\mathcal{A}(\cdot, e)=\left(a^{1}(\cdot), a^{2}(\cdot)\right) \in C^{1}(\bar{\Omega}), \\
\operatorname{div}(\mathcal{A}(X, e)) \geqslant 0 \quad \text { in } C^{0}(\Omega),
\end{gathered}
$$




$$
\begin{gathered}
\Gamma=\partial \Omega \quad \text { is of class } C^{1}, \\
\mathcal{A}(X, e) \cdot v \neq 0 \quad \forall X \in \partial \Omega .
\end{gathered}
$$

Then we consider the following differential system:

$$
(E(\omega, h))\left\{\begin{array}{l}
X^{\prime}(t, \omega, h)=\mathcal{A}(X(t, \omega, h), e) \\
X(0, \omega, h)=(\omega, h)
\end{array}\right.
$$

where $h \in \pi_{y}(\Omega)$ and $\omega \in \pi_{x}(\Omega \cap[y=h])$ and where $\pi_{x}$ and $\pi_{y}$ are respectively the orthogonal projections on the $x$ and $y$ axes.

By the classical theory of ordinary differential equations there exists a unique maximal solution $X(\cdot, \omega, h)$ of $E(\omega, h)$ which is defined on $\left[\alpha_{-}(\omega, h), \alpha_{+}(\omega, h)\right]$ with $X\left(\alpha_{-}(\omega, h), \omega, h\right) \in \partial \Omega \cap$ $[y<h], X\left(\alpha_{+}(\omega, h), \omega, h\right) \in \partial \Omega \cap[y>h]$ (see Figure 2).

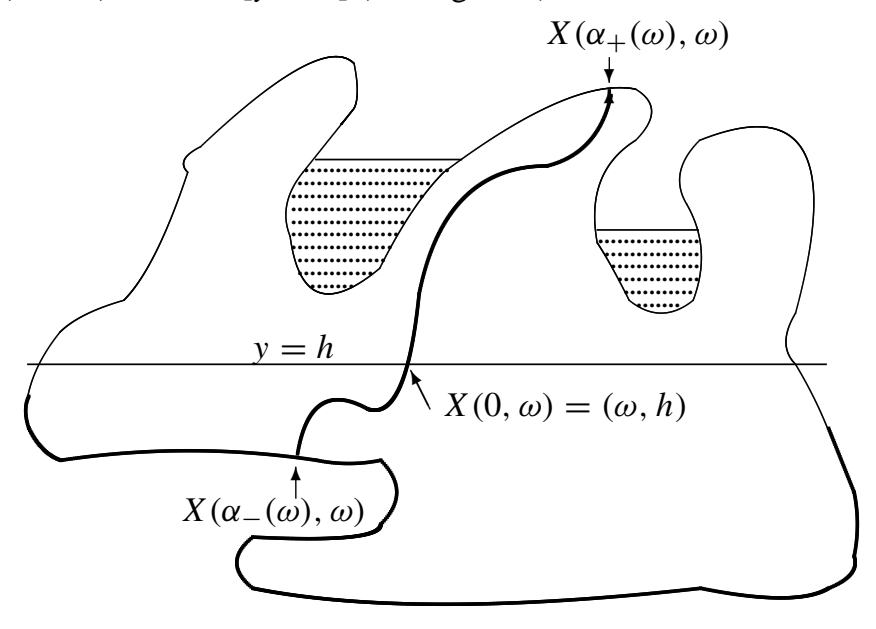

FIG. 2

For simplicity we will denote $X(t, \omega, h), \alpha_{-}(\omega, h)$ and $\alpha_{+}(\omega, h)$ respectively by $X(t, \omega)$, $\alpha_{-}(\omega)$ and $\alpha_{+}(\omega)$. We note that (2.4) means that the orbits of $E(\omega, h)$ do not meet $\partial \Omega$ tangentially. Moreover under the assumptions (2.1), (2.3) and (2.4), one has

Proposition $2.1 \alpha_{-}, \alpha_{+} \in C^{1}\left(\pi_{x}(\Omega \cap[y=h])\right)$.

Proof. Let $h \in \pi_{y}(\Omega)$ and $\omega_{0} \in \pi_{x}(\Omega \cap[y=h])$. By (2.3) there exists a $C^{1}$ function $\sigma$ and $\eta>0$ small enough such that one of the following situations holds:

$$
\begin{array}{lll}
\text { (i) } & \sigma\left(X_{1}\left(\alpha_{-}(\omega), \omega\right)\right)=X_{2}\left(\alpha_{-}(\omega), \omega\right) & \forall \omega \in\left(\omega_{0}-\eta, \omega_{0}+\eta\right), \\
\text { (ii) } & \sigma\left(X_{2}\left(\alpha_{-}(\omega), \omega\right)\right)=X_{1}\left(\alpha_{-}(\omega), \omega\right) & \forall \omega \in\left(\omega_{0}-\eta, \omega_{0}+\eta\right) .
\end{array}
$$

Assume for example that (i) holds. This means that $\alpha_{-}(\omega)$ satisfies

$$
F\left(\alpha_{-}(\omega), \omega\right)=0 \quad \forall \omega \in\left(\omega_{0}-\eta, \omega_{0}+\eta\right), \quad \text { with } \quad F=\sigma \circ X_{1}-X_{2} .
$$

Taking into account $(2.1)$ there exists an open set $\Omega^{*}$ containing $\bar{\Omega}$ such that $\mathcal{A}(\cdot, e) \in C^{1}\left(\Omega^{*}\right)$. Then for each $\omega \in \pi_{x}\left(\Omega^{*} \cap[y=h]\right)$, there exists a unique maximal solution $X^{*}(\cdot, \omega)$ of the 
differential system $E(\omega, h)$ defined on $\left[\alpha_{-}^{*}(\omega), \alpha_{+}^{*}(\omega)\right]$. Obviously we have $X_{\mid\left(\alpha_{-}(\omega), \alpha_{+}(\omega)\right)}^{*}=X$ when $\omega \in \pi_{x}(\Omega \cap[y=h])$.

Let $F^{*}=\sigma \circ X_{1}^{*}-X_{2}^{*}$ defined on $D^{*}=\left\{(t, \omega) \mid \omega \in\left(\omega_{0}-\eta, \omega_{0}+\eta\right), t \in\left(\alpha_{-}^{*}(\omega), \alpha_{+}^{*}(\omega)\right)\right\}$. We have $F^{*} \in C^{1}\left(D^{*}\right)$ since $X_{i}^{*} \in C^{1}\left(D^{*}\right)$ and $\sigma$ is $C^{1}$. In addition $F^{*}$ is a $C^{1}$ extension of $F$ to $D^{*}$ and by (2.1) we have

$$
\begin{aligned}
\frac{\partial F^{*}}{\partial t}(t, \omega) & =\sigma^{\prime}\left(X_{1}^{*}(t, \omega)\right) \cdot \frac{\partial X_{1}^{*}}{\partial t}(t, \omega)-\frac{\partial X_{2}^{*}}{\partial t}(t, \omega) \\
& =\sigma^{\prime}\left(X_{1}^{*}(t, \omega)\right) \cdot a^{1}\left(X^{*}(t, \omega)\right)-a^{2}\left(X^{*}(t, \omega)\right) .
\end{aligned}
$$

In particular by (2.4) we obtain

$$
\frac{\partial F^{*}}{\partial t}\left(\alpha_{-}\left(\omega_{0}\right), \omega_{0}\right)=\sigma^{\prime}\left(X_{1}\left(\alpha_{-}\left(\omega_{0}\right), \omega_{0}\right)\right) \cdot a^{1}\left(X\left(\alpha_{-}\left(\omega_{0}\right), \omega_{0}\right)\right)-a^{2}\left(X\left(\alpha_{-}\left(\omega_{0}\right), \omega_{0}\right)\right) \neq 0 .
$$

Therefore by the implicit function theorem, we deduce that there exists $\delta \in(0, \eta)$ and a unique function $f:\left(\omega_{0}-\delta, \omega_{0}+\delta\right) \rightarrow \mathbb{R}$ such that

$$
F^{*}(t, \omega)=0 \Leftrightarrow t=f(\omega), \quad f\left(\omega_{0}\right)=\alpha_{-}\left(\omega_{0}\right), \quad f \in C^{1}\left(\omega_{0}-\delta, \omega_{0}+\delta\right) .
$$

As $F^{*}\left(\alpha_{-}(\omega), \omega\right)=F\left(\alpha_{-}(\omega), \omega\right)=0$, it follows that $\alpha_{-}(\omega)=f(\omega)$ and $\alpha_{-} \in C^{1}\left(\omega_{0}-\delta, \omega_{0}+\delta\right)$.

If (ii) holds, the proof is similar. Thus $\alpha_{-} \in C^{1}\left(\pi_{x}(\Omega \cap[y=h])\right)$. In the same way we prove that $\alpha_{+} \in C^{1}\left(\pi_{x}(\Omega \cap[y=h])\right)$.

DEFinition 2.1 For each $h \in \pi_{y}(\Omega)$ we define the set

$$
D_{h}=\left\{(t, \omega) \mid \omega \in \pi_{x}(\Omega \cap[y=h]), t \in\left(\alpha_{-}(\omega), \alpha_{+}(\omega)\right)\right\}
$$

and consider the mappings $T_{h}: D_{h} \rightarrow T_{h}\left(D_{h}\right)$ and $S_{h}: D_{h} \rightarrow S_{h}\left(D_{h}\right)$ defined by

$$
T_{h}(t, \omega)=X(t, \omega)=\left(T_{h}^{1}, T_{h}^{2}\right)(t, \omega), \quad S_{h}(t, \omega)=\left(\omega, L_{h}(t, \omega)\right)=(\omega, \tau),
$$

where

$$
L_{h}(t, \omega)=\int_{\alpha_{-}(\omega)}^{t}|\mathcal{A}(X(s, \omega), e)| \mathrm{d} s=\int_{\alpha_{-}(\omega)}^{t}\left|X^{\prime}(s, \omega)\right| \mathrm{d} s
$$

represents the arc length of the curve $X(\cdot, \omega)$ from the point $X\left(\alpha_{-}(\omega), \omega\right)$ to $X(t, \omega)$.

Then we have

PROPOSITION 2.2

$$
\Omega=\bigsqcup_{h \in \pi_{y}(\Omega)} T_{h}\left(D_{h}\right), \quad T_{h} \text { and } S_{h} \text { are } C^{1} \text { diffeomorphisms. }
$$

Proof. First for each $(x, y) \in \Omega$ we have $(x, y)=X(0, \omega)=T_{h}(0, \omega)$ with $\omega=x$ and $h=y$. Next thanks to (2.1) we have $T_{h} \in C^{1}\left(D_{h}\right)$. By Proposition 2.1, $S_{h}$ is also in $C^{1}\left(D_{h}\right)$. To see that they are diffeomorphisms, it suffices to verify that $\operatorname{det}\left(\mathcal{J} T_{h}\right)$ and $\operatorname{det}\left(\mathcal{J} S_{h}\right)$ do not vanish; here we denote by $\mathcal{J} F$ the Jacobian matrix of the transformation $F$. 
One can easily check that

$$
\begin{aligned}
& \operatorname{det} \mathcal{J} S_{h}=-|\mathcal{A}(X(t, \omega), e)|<0, \\
& Y_{h}(t, \omega)=\operatorname{det}\left(\mathcal{J} T_{h}\right)=a^{1}(X(t, \omega)) \frac{\partial X_{2}}{\partial \omega}-a^{2}(X(t, \omega)) \frac{\partial X_{1}}{\partial \omega}, \\
& \frac{\partial Y_{h}}{\partial t}(t, \omega)=Y_{h}(t, \omega) \cdot\{\operatorname{div}(\mathcal{A}(\cdot, e))\}(X(t, \omega)) .
\end{aligned}
$$

Therefore

$$
Y_{h}(t, \omega)=Y_{h}(0, \omega) \exp \left(\int_{0}^{t}\{\operatorname{div}(\mathcal{A}(\cdot, e))\}(X(s, \omega)) \mathrm{d} s\right) .
$$

Since $Y_{h}(0, \omega)=-a^{2}(X(0, \omega))<0$, we get $Y_{h}(t, \omega)<0$ for all $t \in\left(\alpha_{-}(\omega), \alpha_{+}(\omega)\right)$ and all $\omega \in \pi_{x}(\Omega \cap[y=h])$.

The following key theorem generalizes the fact that $g_{y} \geqslant 0$ in $\mathcal{D}^{\prime}(\Omega)$ when $a_{1}=0$ and $a_{2}$ is nondecreasing with respect to $y$ (see [15], [17], and [27]). It will play a major role for the definition of the free boundary and the proof of its continuity.

THEOREM 2.1 Let $(u, g)$ be a solution of $(\mathrm{P})$. For each $h \in \pi_{y}(\Omega)$,

$$
\frac{\partial}{\partial \tau}\left(\widetilde{g} \cdot\left(-Y_{h} \circ S_{h}^{-1}\right)\right) \geqslant 0 \quad \text { in } \mathcal{D}^{\prime}\left(S_{h}\left(D_{h}\right)\right),
$$

where $Y_{h}$ is given by (2.5) and $\widetilde{g}=g \circ T_{h} \circ S_{h}^{-1}$.

Proof. Let $\phi \in \mathcal{D}\left(S_{h}\left(D_{h}\right)\right), \phi \geqslant 0$. Then $\phi \circ S_{h} \circ T_{h}^{-1} \in C_{0}^{1}\left(T_{h}\left(D_{h}\right)\right)$ and by (1.5) and (2.2), we have

$$
\int_{T_{h}\left(D_{h}\right)} g \mathcal{A}(X, e) \nabla\left(\phi \circ S_{h} \circ T_{h}^{-1}\right) \mathrm{d} X \leqslant 0 .
$$

Using the change of variables $T_{h}(t, \omega)=(x, y)$ and the fact that

$$
\mathcal{A}(X(t, \omega), e)\left(\nabla\left(\phi \circ S_{h} \circ T_{h}^{-1}\right)\right) \circ T_{h} \cdot\left(-Y_{h}(t, \omega)\right)=-Y_{h}(t, \omega) \frac{\partial}{\partial t}\left(\phi \circ S_{h}\right)
$$

we get

$$
\int_{D_{h}} g \circ T_{h}(t, \omega) \cdot\left(-Y_{h}(t, \omega)\right) \cdot \frac{\partial}{\partial t}\left(\phi \circ S_{h}\right) \mathrm{d} t \mathrm{~d} \omega \leqslant 0,
$$

which becomes, after using the change of variables $S_{h}^{-1}$,

$$
\int_{S_{h}\left(D_{h}\right)} g \circ T_{h} \circ S_{h}^{-1}(\omega, \tau) \cdot\left(-Y_{h} \circ S_{h}^{-1}(\omega, \tau)\right) \cdot\left(\frac{\partial}{\partial t}\left(\phi \circ S_{h}\right)\right) \circ S_{h}^{-1} \cdot\left|\operatorname{det}\left(\mathcal{J} S_{h}^{-1}\right)\right| \mathrm{d} \omega \mathrm{d} \tau \leqslant 0 .
$$

Taking into account that

$$
\left(\frac{\partial}{\partial t}\left(\phi \circ S_{h}\right)\right) \circ S_{h}^{-1}=\frac{\partial \phi}{\partial \tau} \cdot|\mathcal{A}(\cdot, e)| \circ T_{h} \circ S_{h}^{-1}(\omega, \tau)=\frac{\partial \phi}{\partial \tau} \cdot\left|\operatorname{det}\left(\mathcal{J} S_{h}\right)\right|,
$$

we obtain

$$
\int_{S_{h}\left(D_{h}\right)} \tilde{g}(\omega, \tau) \cdot\left(-Y_{h} \circ S_{h}^{-1}(\omega, \tau)\right) \cdot \frac{\partial \phi}{\partial \tau} \mathrm{d} \omega \mathrm{d} \tau \leqslant 0
$$


REMARK 2.1 In order to avoid complicated notations we will write $\tilde{f}$ to denote the function $f \circ$ $T_{h} \circ S_{h}^{-1}$ for any function $f$ defined on $T_{h}\left(D_{h}\right)$. We will also denote by $\mathcal{T}_{h}$ and $\mathcal{Y}_{h}$ the functions $T_{h} \circ S_{h}^{-1}$ and $-Y_{h} \circ S_{h}^{-1}$ respectively.

\section{Lower semicontinuity of the free boundary}

In what follows we assume that there exist nonnegative constants $\kappa, \sigma$ and positive constants $\lambda_{0}, \lambda_{1}$ with $\sigma \leqslant 1$ and $\lambda_{1} \geqslant \lambda_{0}$ such that for all $X, Y \in \bar{\Omega}, \zeta, \xi \in \mathbb{R}^{2}$,

$$
\begin{gathered}
\sum_{i, j} \frac{\partial \mathcal{A}^{i}}{\partial \zeta_{j}}(X, \zeta) \xi_{i} \xi_{j} \geqslant \lambda_{0}\left(\kappa+|\zeta|^{q-2}\right)|\xi|^{2}, \\
\left|\frac{\partial \mathcal{A}^{i}}{\partial \zeta_{j}}(X, \zeta)\right| \leqslant \lambda_{1}\left(\kappa+|\zeta|^{q-2}\right), \\
|\mathcal{A}(X, \zeta)-\mathcal{A}(Y, \zeta)| \leqslant \lambda_{1}\left(1+|\zeta|^{q-1}\right)\left(|X-Y|^{\sigma}\right) .
\end{gathered}
$$

REMARK $3.1 \quad$ (i) If $\mathcal{A}(X, \zeta)=a(X) \zeta$ with $a(X)$ a bounded 2-by-2 matrix, then (3.1) and (3.2) are satisfied and (3.3) is not needed. In this case $u \in C_{\text {loc }}^{0, \gamma}\left(\Omega \cup S_{2} \cup S_{3}\right)$ for some $\gamma \in(0,1)$.

(ii) Assumptions (3.1)-(3.3) are satisfied in the case where $\mathcal{A}(X, \zeta)=|\zeta|^{q-2} \zeta$. Moreover under these assumptions, we deduce from (1.4) (see [20], [30]) that $u \in C_{\text {loc }}^{0, \gamma}\left(\Omega \cup S_{2} \cup S_{3}\right)$ for some $\gamma \in(0,1)$. Also by $(1.4)$ and $(\mathrm{P})(\mathrm{i})$ we have $\operatorname{div}(\mathcal{A}(X, u))=0$ in $\mathcal{D}^{\prime}([u>y])$. It then follows by (3.1)-(3.3) (see [19], [25] for example) that $u \in C_{\text {loc }}^{1, \delta}([u>y])$ for some $\delta \in(0,1)$.

The following strong maximum principle (see [18], [1]) will be needed:

LEMMA 3.1 (Strong maximum principle) Let $u_{1}$ and $u_{2}$ be two functions defined on a domain $D$ of $\mathbb{R}^{2}$ such that $u_{1}, u_{2} \in C^{1}(D), u_{1} \geqslant u_{2}$ in $D$, the set $\left\{X \in D \mid \nabla u_{1}(X)=\nabla u_{2}(X)=0\right\}$ is empty and $\operatorname{div}\left(\mathcal{A}\left(X, \nabla u_{1}\right)-\mathcal{A}\left(X, \nabla u_{2}\right)\right) \leqslant 0$. Then either

$$
u_{1}=u_{2} \text { in } D \quad \text { or } \quad u_{1}>u_{2} \text { in } D .
$$

The theorem below will allow us to define the free boundary $\partial([u>y]) \cap \Omega$ locally as a curve.

THEOREM 3.1 Let $(u, g)$ be a solution of $(\mathrm{P})$ and $X_{0}=\mathcal{T}_{h}\left(\omega_{0}, \tau_{0}\right)=\left(x_{0}, y_{0}\right) \in \Omega$.

(i) If $p\left(X_{0}\right)=\widetilde{p}\left(\omega_{0}, \tau_{0}\right)>0$, then there exists $\epsilon>0$ such that

$$
\tilde{p}(\omega, \tau)>0 \quad \forall(\omega, \tau) \in C_{\epsilon}=\left\{(\omega, \tau) \in S_{h}\left(D_{h}\right)|| \omega-\omega_{0} \mid<\epsilon, \tau<\tau_{0}+\epsilon\right\},
$$

(ii) If $p\left(X_{0}\right)=\widetilde{p}\left(\omega_{0}, \tau_{0}\right)=0$, then $\widetilde{p}\left(\omega_{0}, \tau\right)=0$ for all $\tau \geqslant \tau_{0}$.

Proof. (i) By continuity, there exists $\epsilon>0$ such that

$$
\tilde{p}(\omega, \tau)>0 \quad \forall(\omega, \tau) \in\left(\omega_{0}-\epsilon, \omega_{0}+\epsilon\right) \times\left(\tau_{0}-\epsilon, \tau_{0}+\epsilon\right)=Q_{\epsilon} .
$$

Then $\widetilde{g}(\omega, \tau)=0$ for a.e. $(\omega, \tau) \in Q_{\epsilon}$. By Theorem 2.1 and since $\mathcal{Y}_{h}>0, \widetilde{g} \geqslant 0$, we get $\widetilde{g}=0$ a.e. in $C_{\epsilon}$, i.e. $g=0$ a.e. in $\mathcal{T}_{h}\left(C_{\epsilon}\right)$ (see Figure 3).

By (1.4) we have $\operatorname{div}(\mathcal{A}(X, \nabla u))=\operatorname{div}(g \mathcal{A}(X, e))=0$ in $\mathcal{D}^{\prime}\left(\mathcal{T}_{h}\left(C_{\epsilon}\right)\right)$. Since $\operatorname{div}(\mathcal{A}(X, \nabla y)) \geqslant 0$ in $\mathcal{D}^{\prime}(\Omega), \nabla y=e \neq 0, u \geqslant y$ in $\mathcal{T}_{h}\left(C_{\epsilon}\right)$ and $u>y$ in $\mathcal{T}_{h}\left(Q_{\epsilon}\right)$, it follows by Lemma 3.1 that $u>y$ in $\mathcal{T}_{h}\left(C_{\epsilon}\right)$.

(ii) This is a consequence of (i). 


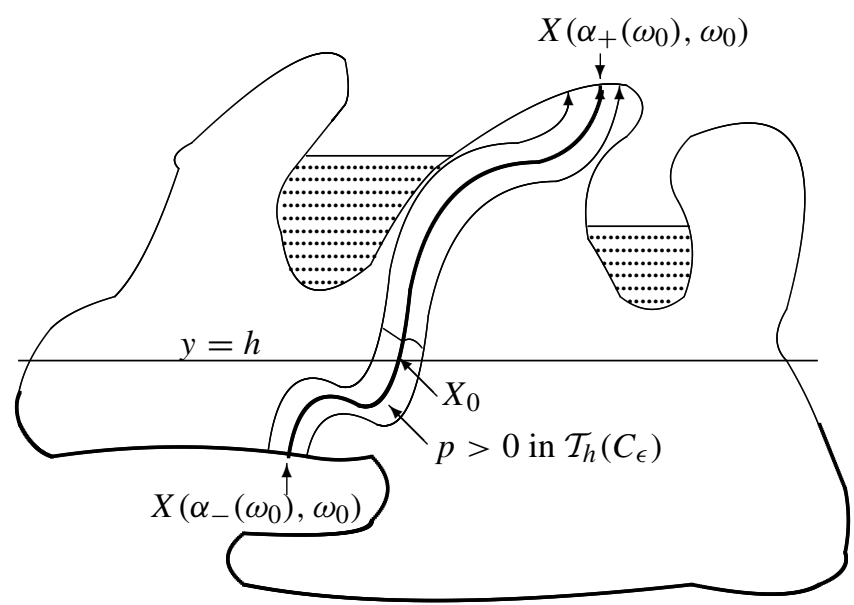

FIG. 3

REMARK 3.2 (i) The result of Theorem 3.1 means that if a point $X_{0}$ is in the wet region, then the part of the curve $X(\cdot, \omega)$ passing through $X_{0}$ at $t_{0}$ remains in the wet region for all $t \leqslant t_{0}$.

(ii) In [17] and [27] we assumed that $\mathcal{A}(X, e)=k(X) e$, which leads to $X_{1}^{\prime}(t)=0$ for all $t$ and the curve $X(\cdot, \omega)$ is a vertical segment. Therefore the free boundary is represented by a curve of the form $y=\Phi(x)$.

(iii) We have $u=p+y=\varphi+y>y$ on $S_{3, i}\left(\varphi>0\right.$ on $\left.S_{3}\right), i=1, \ldots, N$, and $u \in C^{0}\left(\Omega \cup S_{3}\right)$. So $p>0$ below $S_{3}$ in the following sense:

$$
p(X(t, \omega))>0 \quad \forall t \in\left[\alpha_{-}(\omega), \alpha_{+}(\omega)\right] \text { such that } X\left(\alpha_{+}(\omega), \omega\right) \in S_{3} .
$$

Definition 3.1 For each $h \in \pi_{y}(\Omega)$ we define the function $\Phi_{h}$ on $\pi_{x}(\Omega \cap[y=h])$ by

$$
\Phi_{h}(\omega)= \begin{cases}\sup \left\{\tau \mid(\omega, \tau) \in S_{h}\left(D_{h}\right), \tilde{p}(\omega, \tau)>0\right\} & \text { if this set is not empty } \\ 0 & \text { otherwise. }\end{cases}
$$

Thanks to Theorem 3.1, $\Phi_{h}$ is well defined and we have

Proposition $3.1 \Phi_{h}$ is lower semicontinuous on $\pi_{x}(\Omega \cap[y=h])$. Moreover

$$
[\tilde{p}(\omega, \tau)>0]=\left[\tau<\Phi_{h}(\omega)\right] .
$$

Proof. First we show the lower semicontinuity of $\Phi_{h}$. Let $\omega_{0} \in \pi_{x}(\Omega \cap[y=h])$.

If $\Phi_{h}\left(\omega_{0}\right)=0$ then for each $\epsilon>0, \Phi_{h}(\omega) \geqslant 0>\Phi_{h}\left(\omega_{0}\right)-\epsilon$ for all $\omega$.

If $\Phi_{h}\left(\omega_{0}\right)>0$ then for each $\epsilon>0$, there is $\tau_{\epsilon}=L_{h}\left(t_{\epsilon}, \omega_{0}\right)>0$ with $t_{\epsilon} \in\left(\alpha_{-}\left(\omega_{0}\right), \alpha_{+}\left(\omega_{0}\right)\right)$ such that

$$
\Phi_{h}\left(\omega_{0}\right) \geqslant \tau_{\epsilon}>\Phi_{h}\left(\omega_{0}\right)-\epsilon / 2, \quad \tilde{p}\left(\omega_{0}, \tau_{\epsilon}\right)>0 .
$$

Moreover (see [23, Theorem 3.4, p. 24]) one can find $\eta_{1}>0$ such that $X(t, \omega)$ exists for all $(t, \omega) \in$ $\left[\alpha_{-}\left(\omega_{0}\right), \alpha_{+}\left(\omega_{0}\right)\right] \times\left(\omega_{0}-\eta_{1}, \omega_{0}+\eta_{1}\right)$ and $(t, \omega) \mapsto X(t, \omega)$ is continuous. Then by continuity, there exists $0<\eta_{2}<\eta_{1}$ such that

$$
p(X(t, \omega))>0 \quad \forall(t, \omega) \in\left(t_{\epsilon}-\eta_{2}, t_{\epsilon}+\eta_{2}\right) \times\left(\omega_{0}-\eta_{2}, \omega_{0}+\eta_{2}\right) .
$$


By Theorem 3.1, we deduce that

$$
p(X(t, \omega))>0 \quad \forall(t, \omega) \in\left(\alpha_{-}(\omega), t_{\epsilon}+\eta_{2}\right) \times\left(\omega_{0}-\eta_{2}, \omega_{0}+\eta_{2}\right) .
$$

Using the definition of $\Phi_{h}$ we get, for $\omega \in\left(\omega_{0}-\eta_{2}, \omega_{0}+\eta_{2}\right)$,

$$
\begin{aligned}
\Phi_{h}(\omega) & =\sup _{t \in\left(\alpha_{-}(\omega), \alpha_{+}(\omega)\right)}\left\{L_{h}(t, \omega) \mid(t, \omega) \in D_{h} \text { and } p(X(t, \omega))>0\right\} \\
& \geqslant L_{h}\left(t_{\epsilon}, \omega\right) .
\end{aligned}
$$

Since $L_{h}\left(t_{\epsilon}, \omega\right)=\int_{\alpha_{-}(\omega)}^{t_{\epsilon}}|\mathcal{A}(X(s, \omega), e)| \mathrm{d} s$ is continuous with respect to $\omega$, there exists $0<\eta<\eta_{2}$ such that for all $\omega \in\left(\omega_{0}-\eta, \omega_{0}+\eta\right)$,

$$
\begin{aligned}
L_{h}\left(t_{\epsilon}, \omega\right) & =\int_{\alpha_{-}(\omega)}^{t_{\epsilon}}|\mathcal{A}(X(s, \omega), e)| \mathrm{d} s \geqslant \int_{\alpha_{-}\left(\omega_{0}\right)}^{t_{\epsilon}}\left|\mathcal{A}\left(X\left(s, \omega_{0}\right), e\right)\right| \mathrm{d} s-\frac{\epsilon}{2} \\
& =L_{h}\left(t_{\epsilon}, \omega_{0}\right)-\frac{\epsilon}{2}=\tau_{\epsilon}-\frac{\epsilon}{2}>\Phi_{h}\left(\omega_{0}\right)-\frac{\epsilon}{2}-\frac{\epsilon}{2} .
\end{aligned}
$$

Thus $\Phi_{h}(\omega) \geqslant \Phi_{h}\left(\omega_{0}\right)-\epsilon$ for all $\omega \in\left(\omega_{0}-\eta, \omega_{0}+\eta\right)$.

Now we prove (3.5). Let $\left(\omega_{0}, \tau_{0}\right) \in\left[\tau<\Phi_{h}(\omega)\right]$. Assume that $\widetilde{p}\left(\omega_{0}, \tau_{0}\right)=0$. Then by Theorem 3.1, $\tilde{p}\left(\omega_{0}, \tau\right)=0$ for all $\tau \geqslant \tau_{0}$. So $\Phi_{h}\left(\omega_{0}\right)=\sup \left\{\tau \mid\left(\omega_{0}, \tau\right) \in S_{h}\left(D_{h}\right)\right.$ and $\left.\tilde{p}\left(\omega_{0}, \tau\right)>0\right\} \leqslant \tau_{0}$, which is a contradiction.

Now let $\left(\omega_{0}, \tau_{0}\right) \in[\tilde{p}(\omega, \tau)>0]$. By continuity, there exists $\eta>0$ such that $\tilde{p}\left(\omega_{0}, \tau\right)>0$ for all $\tau \in\left(\tau_{0}-\eta, \tau_{0}+\eta\right)$. By Theorem 3.1, we deduce that $\tilde{p}\left(\omega_{0}, \tau\right)>0$ for all $\tau<\tau_{0}+\eta$ such that $\left(\omega_{0}, \tau\right) \in S_{h}\left(D_{h}\right)$. Hence $\Phi_{h}\left(\omega_{0}\right) \geqslant \tau_{0}+\eta>\tau_{0}$ and $\left(\omega_{0}, \tau_{0}\right) \in\left[\tau<\Phi_{h}(\omega)\right]$.

\section{Some technical lemmas}

The following lemma plays an important role in the proof of the continuity of the free boundary.

LEMMA 4.1 Let $(u, g)$ be a solution of $(\mathrm{P})$. Let $\left(\omega_{1}, \tau_{0}\right),\left(\omega_{2}, \tau_{0}\right) \in S_{h}\left(D_{h}\right)$ with $\omega_{1}<\omega_{2}$ and

$$
\tilde{p}\left(\omega_{i}, \tau\right)=0 \quad \forall\left(\omega_{i}, \tau\right) \in S_{h}\left(D_{h}\right), \tau>\tau_{0} \geqslant 0 .
$$

Set $Z_{\tau_{0}}=\mathcal{T}_{h}\left(\left(\omega_{1}, \omega_{2}\right) \times\left(\tau_{0},+\infty\right) \cap S_{h}\left(D_{h}\right)\right)$ and assume that $\bar{Z}_{\tau_{0}} \cap S_{3}=\emptyset$. Let $y_{0} \in \mathbb{R}$ be such that $D_{y_{0}, \tau_{0}}=\left[y>y_{0}\right] \cap Z_{\tau_{0}} \neq \varnothing$ (see Figure 4). Then

$$
\int_{D_{y_{0}, \tau_{0}}}(\mathcal{A}(X, \nabla u)-g \mathcal{A}(X, e)) \cdot e \mathrm{~d} X \leqslant 0 .
$$

To prove this lemma, we need another lemma:

LEMMA 4.2 Under the assumptions of Lemma 4.1, we have

$$
\int_{D_{y_{0}, \tau_{0}}}(\mathcal{A}(X, \nabla u)-\chi([u=y]) \mathcal{A}(X, e)) \cdot \nabla \zeta \mathrm{d} X \leqslant \int_{\omega_{1}}^{\omega_{2}} \mathcal{Y}_{h}\left(s, \Phi_{h}(s)\right) \cdot \widetilde{\zeta}\left(s, \Phi_{h}(s)\right) \mathrm{d} s
$$

for all $\zeta \in W^{1, q}\left(D_{y_{0}, \tau_{0}}\right) \cap C^{0}\left(\bar{D}_{y_{0}, \tau_{0}}\right)$ such that $\zeta \geqslant 0$ and $\zeta\left(x, y_{0}\right)=0$ for all $\left(x, y_{0}\right) \in \bar{D}_{y_{0}, \tau_{0}}$. 


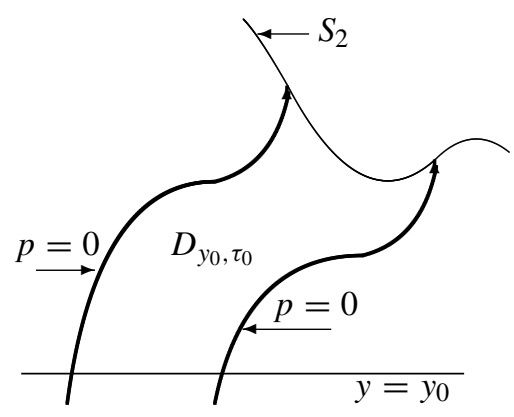

FIG. 4

Proof of Lemma 4.2. For $\epsilon>0, \xi=\chi\left(D_{y_{0}, \tau_{0}}\right) \min ((u-y) / \epsilon, \zeta)$ is a test function for (P). So since $g \cdot(u-y)=0$ a.e. in $\Omega$, we have

$$
\int_{D_{y_{0}, \tau_{0}}} \mathcal{A}(X, \nabla u) \cdot \nabla\left(\frac{u-y}{\epsilon} \wedge \zeta\right) \mathrm{d} X \leqslant 0 .
$$

Using the monotonicity of $\mathcal{A}$, we get

$$
\begin{aligned}
\int_{D_{y_{0}, \tau_{0}} \cap[u-y \geqslant \epsilon \zeta]}(\mathcal{A}(X, \nabla u)-\mathcal{A}(X, e)) \cdot \nabla \zeta \mathrm{d} X & \leqslant-\int_{D_{y_{0}, \tau_{0}}} \mathcal{A}(X, e) \cdot \nabla\left(\frac{u-y}{\epsilon} \wedge \zeta\right) \mathrm{d} X \\
& =-I_{\epsilon}
\end{aligned}
$$

Moreover

$$
\begin{aligned}
I_{\epsilon}= & \int_{D_{y_{0}, \tau_{0}}} \chi([u>y]) \mathcal{A}(X, e) \cdot \nabla\left(\frac{u-y}{\epsilon} \wedge \zeta\right) \mathrm{d} X \\
= & \int_{D_{y_{0}, \tau_{0}}} \chi([u>y]) \mathcal{A}(X, e) \cdot \nabla \zeta \mathrm{d} X \\
& -\int_{D_{y_{0}, \tau_{0}}} \mathcal{A}(X, e) \cdot \nabla\left(\zeta-\frac{u-y}{\epsilon}\right)^{+} \chi([u>y]) \mathrm{d} X=I^{1}-I_{\epsilon}^{2} .
\end{aligned}
$$

Now using the change of variables $T_{h}$, we obtain

$$
\begin{aligned}
I_{\epsilon}^{2} & =\int_{T_{h}^{-1}\left(D_{\left.y_{0}, \tau_{0}\right)}\right.} \chi\left(\left[p \circ T_{h}(t, \omega)>0\right]\right) \mathcal{A}(X(t, \omega), e) \cdot\left(\nabla\left(\zeta-\frac{p}{\epsilon}\right)^{+}\right) \circ T_{h}\left|Y_{h}(t, \omega)\right| \mathrm{d} t \mathrm{~d} \omega \\
& =-\int_{T_{h}^{-1}\left(D_{y_{0}, \tau_{0}}\right)} \chi\left(\left[p \circ T_{h}(t, \omega)>0\right]\right) \cdot Y_{h}(t, \omega) \cdot \frac{\partial}{\partial t}\left(\left(\zeta-\frac{p}{\epsilon}\right)^{+} \circ T_{h}\right) \mathrm{d} t \mathrm{~d} \omega
\end{aligned}
$$

since

$$
\mathcal{A}(X(t, \omega), e) \cdot\left(\nabla\left(\zeta-\frac{p}{\epsilon}\right)^{+}\right) \circ T_{h} \cdot\left|Y_{h}(t, \omega)\right|=-Y_{h}(t, \omega) \cdot \frac{\partial}{\partial t}\left(\left(\zeta-\frac{p}{\epsilon}\right)^{+} \circ T_{h}\right) .
$$


Next using the change of variables $S_{h}^{-1}$, we get

$$
\begin{aligned}
I_{\epsilon}^{2}=-\int_{S_{h} \circ T_{h}^{-1}\left(D_{y_{0}, \tau_{0}}\right)} \chi([\tilde{p}(\omega, \tau)>0]) \cdot Y_{h} \circ S_{h}^{-1}(\omega, \tau) \\
\cdot\left(\frac{\partial}{\partial t}\left(\left(\zeta-\frac{p}{\epsilon}\right)^{+} \circ T_{h}\right)\right) \circ S_{h}^{-1}(\omega, \tau) \cdot\left|\operatorname{det}\left(\mathcal{J} S_{h}^{-1}\right)\right| \mathrm{d} \omega \mathrm{d} \tau \\
=\int_{\mathcal{T}_{h}^{-1}\left(D_{y_{0}, \tau_{0}}\right)} \chi([\tilde{p}(\omega, \tau)>0]) \cdot \mathcal{Y}_{h}(\omega, \tau) \cdot \frac{\partial}{\partial \tau}\left(\zeta-\frac{p}{\epsilon}\right)^{+}(\omega, \tau) \mathrm{d} \omega \mathrm{d} \tau
\end{aligned}
$$

since

$$
\left(\frac{\partial}{\partial t}\left(\left(\zeta-\frac{p}{\epsilon}\right)^{+} \circ T_{h}\right)\right) \circ S_{h}^{-1}(\omega, \tau)=\frac{\partial}{\partial \tau}\left(\left(\zeta-\frac{p}{\epsilon}\right)^{+} \circ T_{h} \circ S_{h}^{-1}\right)(\omega, \tau) \cdot\left|\mathcal{A}\left(T_{h} \circ S_{h}^{-1}(\omega, \tau), e\right)\right| .
$$

Therefore

$$
I_{\epsilon}^{2}=\int_{\left(\omega_{1}, \omega_{2}\right) \times\left(\tau_{0},+\infty\right) \cap \mathcal{T}_{h}^{-1}\left(\left[y>y_{0}\right]\right)} \chi\left(\left[\tau<\Phi_{h}(\omega)\right]\right) \cdot \mathcal{Y}_{h} \cdot \frac{\partial}{\partial \tau}\left(\widetilde{\zeta-\frac{p}{\epsilon}}\right)^{+} \mathrm{d} \omega \mathrm{d} \tau .
$$

Note that for every $\omega \in\left(\omega_{1}, \omega_{2}\right)$ there is a unique $t_{y_{0}}(\omega)$ such that

$$
X_{2}\left(t_{y_{0}}(\omega), \omega\right)=y_{0}, \quad \tau_{y_{0}}(\omega)=\int_{\alpha_{-}(\omega)}^{t_{y_{0}}(\omega)}|\mathcal{A}(X(s, \omega), e)| \mathrm{d} s,
$$

and one can check that

$\left(\left(\omega_{1}, \omega_{2}\right) \times\left(\tau_{0},+\infty\right)\right) \cap \mathcal{T}_{h}^{-1}\left(\left[y>y_{0}\right]\right)=\left\{(\omega, \tau) \in S_{h}\left(D_{h}\right) \mid \omega \in\left(\omega_{1}, \omega_{2}\right), \tau>\sup \left(\tau_{0}, \tau_{y_{0}}(\omega)\right)\right\}$.

It follows by the second mean value theorem that

$$
\begin{aligned}
I_{\epsilon}^{2} & =\int_{\omega_{1}}^{\omega_{2}} \int_{\sup \left(\tau_{0}, \tau_{y_{0}}(\omega)\right)}^{\Phi_{h}(\omega)} \mathcal{Y}_{h}(\omega, \tau) \cdot \frac{\partial}{\partial \tau}\left(\widetilde{\zeta-\frac{p}{\epsilon}}\right)^{+}(\omega, \tau) \mathrm{d} \omega \mathrm{d} \tau \\
& =\int_{\omega_{1}}^{\omega_{2}} \mathcal{Y}_{h}\left(\omega, \Phi_{h}(\omega)\right) \int_{\tau^{*}\left(y_{0}, \omega\right)}^{\Phi_{h}(\omega)} \frac{\partial}{\partial \tau}\left(\widetilde{\zeta-\frac{p}{\epsilon}}\right)^{+}(\omega, \tau) \mathrm{d} \tau \\
& \leqslant \int_{\omega_{1}}^{\omega_{2}} \mathcal{Y}_{h}\left(\omega, \Phi_{h}(\omega)\right) \tilde{\zeta}\left(\omega, \Phi_{h}(\omega)\right) \mathrm{d} \omega
\end{aligned}
$$

where $\tau^{*}\left(y_{0}, \omega\right) \in\left[\sup \left(\tau_{0}, \tau_{y_{0}}(\omega)\right), \Phi_{h}(\omega)\right]$. Thus

$$
\begin{aligned}
\int_{D_{y_{0}, \tau_{0}} \cap[u-y \geqslant \epsilon \zeta]}(\mathcal{A}(X, \nabla u)-\mathcal{A}(X, e)) \cdot \nabla \zeta \mathrm{d} X+ & \int_{D_{y_{0}, \tau_{0}}} \chi([u>y]) \mathcal{A}(X, e) \cdot \nabla \zeta \mathrm{d} X \\
& \leqslant \int_{\omega_{1}}^{\omega_{2}} \mathcal{Y}_{h}(\omega, \Phi(\omega)) \cdot \tilde{\zeta}\left(\omega, \Phi_{h}(\omega)\right) \mathrm{d} \omega
\end{aligned}
$$

and the lemma follows by letting $\epsilon$ go to 0 . 
Proof of Lemma 4.1. Let $\epsilon>0$ and $h_{\epsilon}=\theta_{\epsilon} \circ S_{h} \circ T_{h}^{-1}$, where

$$
\theta_{\epsilon}(\omega)=\min \left(\frac{\left(\omega-\omega_{1}\right)^{+}}{\epsilon}, 1\right) \cdot \min \left(\frac{\left(\omega_{2}-\omega\right)^{+}}{\epsilon}, 1\right) .
$$

Since $\chi\left(D_{y_{0}, \tau_{0}}\right) h_{\epsilon}\left(y-y_{0}\right)$ is a test function for $(\mathrm{P})$ we have

$$
\begin{aligned}
& \int_{D_{y_{0}, \tau_{0}}}(\mathcal{A}(X, \nabla u)-g \mathcal{A}(X, e)) \cdot e \mathrm{~d} X \\
&=\int_{D_{y_{0}, \tau_{0}}}(\mathcal{A}(X, \nabla u)-g \mathcal{A}(X, e)) \cdot \nabla\left(y-y_{0}\right) \mathrm{d} X \\
&= \int_{D_{y_{0}, \tau_{0}}}(\mathcal{A}(X, \nabla u)-g \mathcal{A}(X, e)) \cdot \nabla\left(h_{\epsilon}\left(y-y_{0}\right)\right) \mathrm{d} X \\
&+\int_{D_{y_{0}, \tau_{0}}}(\mathcal{A}(X, \nabla u)-g \mathcal{A}(X, e)) \cdot \nabla\left(\left(1-h_{\epsilon}\right)\left(y-y_{0}\right)\right) \mathrm{d} X \\
& \leqslant \int_{D_{y_{0}, \tau_{0}}}(\mathcal{A}(X, \nabla u)-g \mathcal{A}(X, e)) \cdot \nabla\left(\left(1-h_{\epsilon}\right)\left(y-y_{0}\right)\right) \mathrm{d} X \\
&= \int_{D_{y_{0}, \tau_{0}}}(\mathcal{A}(X, \nabla u)-\chi([u=y]) \mathcal{A}(X, e)) \cdot \nabla\left(\left(1-h_{\epsilon}\right)\left(y-y_{0}\right)\right) \mathrm{d} X \\
&+\int_{D_{y_{0}, \tau_{0}}}(\chi([u=y])-g) \mathcal{A}(X, e) \cdot \nabla\left(\left(1-h_{\epsilon}\right)\left(y-y_{0}\right)\right) \mathrm{d} X=J_{\epsilon}^{1}+J_{\epsilon}^{2} .
\end{aligned}
$$

By Lemma 4.2, with $\zeta=\left(1-h_{\epsilon}\right) \cdot\left(y-y_{0}\right)=\left(1-\theta_{\epsilon} \circ S_{h} \circ T_{h}^{-1}\right) \cdot\left(y-y_{0}\right)$, we have

$$
J_{\epsilon}^{1} \leqslant \int_{\omega_{1}}^{\omega_{2}} \mathcal{Y}_{h}\left(\omega, \Phi_{h}(\omega)\right) \cdot\left(1-\theta_{\epsilon}(\omega)\right) \cdot\left(\widetilde{y-y_{0}}\right)\left(\omega, \Phi_{h}(\omega)\right) \mathrm{d} \omega .
$$

Moreover

$$
\begin{aligned}
& J_{\epsilon}^{2}= \int_{\mathcal{T}_{h}^{-1}\left(D_{y_{0}, \tau_{0}}\right)}(\chi([\tilde{p}(\omega, \tau)=0])-\tilde{g}(\omega, \tau)) \\
& \cdot \mathcal{Y}_{h}(\omega, \tau) \cdot \frac{\partial}{\partial \tau}\left(\left(1-\theta_{\epsilon}(\omega)\right) \cdot\left(\widetilde{y-y_{0}}\right)(\omega, \tau)\right) \mathrm{d} \omega \mathrm{d} \tau \\
&=\int_{\mathcal{T}_{h}^{-1}\left(D_{y_{0}, \tau_{0}}\right)}(\chi([\tilde{p}(\omega, \tau)=0])-\tilde{g}(\omega, \tau)) \\
& \cdot \mathcal{Y}_{h}(\omega, \tau) \cdot\left(1-\theta_{\epsilon}(\omega)\right) \frac{\partial}{\partial \tau}\left(T_{h}^{2} \circ S_{h}^{-1}(\omega, \tau)\right) \mathrm{d} \omega \mathrm{d} \tau .
\end{aligned}
$$

Since $\theta_{\epsilon} \rightarrow 1$ as $\epsilon \rightarrow 0$, we conclude that $J_{\epsilon}^{1}+J_{\epsilon}^{2} \rightarrow 0$. This completes the proof.

LEMMA 4.3 Let $(u, g)$ be a solution of $(\mathrm{P})$. Let $\mathcal{C}_{h}$ be the connected component of $\left[\tau<\Phi_{h}(\omega)\right]$ such that $\overline{\mathcal{T}_{h}\left(\mathcal{C}_{h}\right)} \cap S_{3}=\emptyset$. Then for $C_{h}=\mathcal{T}_{h}\left(\mathcal{C}_{h}\right)$ we have

$$
\int_{C_{h}}(\mathcal{A}(X, \nabla u)-g \mathcal{A}(X, e)) \cdot e \mathrm{~d} X \leqslant 0 .
$$


Proof. Step 1. Arguing as in the proof of Lemma 4.2, we get, for all nonnegative $\zeta \in W^{1, q}\left(\mathcal{C}_{h}\right) \cap$ $C^{0}\left(\overline{\mathcal{C}}_{h}\right)$,

$$
\int_{C_{h}}(\mathcal{A}(X, \nabla u)-\chi([u=y]) \mathcal{A}(X, e)) \cdot \nabla \zeta \mathrm{d} X \leqslant \int_{\pi_{\omega}\left(\mathcal{C}_{h}\right)} \mathcal{Y}_{h}\left(s, \Phi_{h}(s)\right) \cdot \tilde{\zeta}\left(s, \Phi_{h}(s)\right) \mathrm{d} s .
$$

Step 2. Let $\epsilon>0$ and $A=\mathbb{R} \backslash \pi_{\omega}\left(\mathcal{C}_{h}\right)$. We consider $\alpha_{\epsilon}(\omega)=\min (1, d(\omega, A) / \epsilon), h_{\epsilon}=\alpha_{\epsilon} \circ \mathcal{T}_{h}^{-1}$ and we argue as in the proof of Lemma 4.1.

Lemma 4.4 Let $(u, g)$ be a solution of (P). Let $X_{0}=\left(x_{0}, y_{0}\right)=\mathcal{T}_{h}\left(\omega_{0}, \tau_{0}\right)$ be a point in $\Omega$, $\left(\omega_{0}, \tau_{0}\right) \in S_{h}\left(D_{h}\right)$. Denote by $B_{r}\left(\omega_{0}, \tau_{0}\right)$ a ball with center $\left(\omega_{0}, \tau_{0}\right)$ and radius $r$ contained in $S_{h}\left(D_{h}\right)$. If $\tilde{p}=0$ in $B_{r}\left(\omega_{0}, \tau_{0}\right)$, then (see Figure 5)

$$
\tilde{p}=0 \quad \text { in } C_{r}, \quad \tilde{g}=1 \quad \text { a.e. in } C_{r},
$$

where $C_{r}=\left\{(\omega, \tau) \in S_{h}\left(D_{h}\right)|| \omega-\omega_{0} \mid<r, \tau>\tau_{0}\right\} \cup B_{r}\left(\omega_{0}, \tau_{0}\right)$, i.e. if $p=0$ in $\mathcal{T}_{h}\left(B_{r}\left(\omega_{0}, \tau_{0}\right)\right)$, then $p=0, g=1$ a.e. in $\mathcal{T}_{h}\left(C_{r}\right)$.

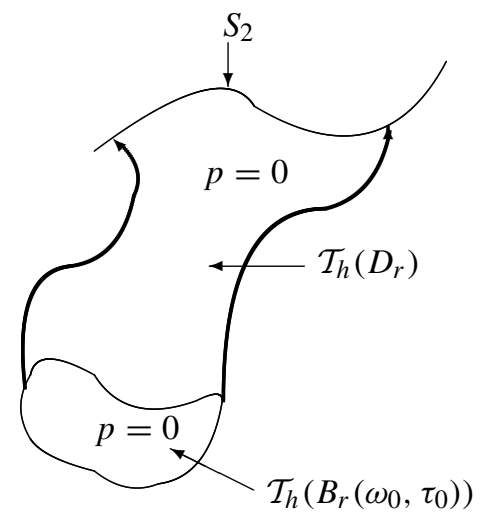

FIG. 5

Proof. Note that by Remark 3.2, we necessarily have

$$
X\left(\alpha_{+}(\omega), \omega\right) \in S_{2} \quad \forall \omega \in\left(\omega_{0}-r, \omega_{0}+r\right) .
$$

By Theorem 3.1(ii), we have $\widetilde{p}=0$ in $C_{r}$. Applying Lemma 4.1 with $Z_{\tau_{0}}=\mathcal{T}_{h}\left(\left(\omega_{1}, \omega_{2}\right) \times\right.$ $\left.\left(\tau_{0},+\infty\right) \cap S_{h}\left(D_{h}\right)\right) \subset \mathcal{T}_{h}\left(C_{r}\right)$ we obtain

$$
\int_{\left[y>y_{0}\right] \cap Z_{\tau_{0}}}(1-g) \mathcal{A}(X, e) \cdot e \mathrm{~d} X \leqslant 0 \quad \forall y_{0} \in \mathbb{R} \text { such that }\left[y>y_{0}\right] \cap Z_{\tau_{0}} \neq \emptyset .
$$

So $g=1$ a.e. in $Z_{\tau_{0}}$. This holds for all domains $Z_{\tau_{0}}$ in $\mathcal{T}_{h}\left(C_{r}\right)$ and we get $g=1$ a.e. in $\mathcal{T}_{h}\left(C_{r}\right)$.

The following result is a sort of maximum principle. 
LEMMA 4.5 Let $(u, g)$ be a solution of $(\mathrm{P}), X_{0}=\left(x_{0}, y_{0}\right)=\mathcal{T}_{h}\left(\omega_{0}, \tau_{0}\right)$ be a point of $\Omega$ and $B_{r}$ be an open ball in $S_{h}\left(D_{h}\right)$ with center $\left(\omega_{0}, \tau_{0}\right)$ and radius $r$. Then the following situations are impossible (see Figures 6, 7 and 8):

$$
\begin{aligned}
& \text { (i) } \begin{cases}\tilde{p}\left(\omega_{0}, \tau\right)=0 & \forall \tau \in\left(\tau_{0}-r, \tau_{0}+r\right), \\
\tilde{p}(\omega, \tau)>0 & \forall(\omega, \tau) \in B_{r} \backslash S, S=\left\{\omega_{0}\right\} \times\left(\tau_{0}-r, \tau_{0}+r\right),\end{cases} \\
& \text { (ii) } \begin{cases}\tilde{p}(\omega, \tau)=0 & \forall(\omega, \tau) \in B_{r} \cap\left[\omega \leqslant \omega_{0}\right], \\
\tilde{p}(\omega, \tau)>0 & \forall(\omega, \tau) \in B_{r} \cap\left[\omega>\omega_{0}\right],\end{cases} \\
& \text { (iii) } \begin{cases}\tilde{p}(\omega, \tau)=0 & \forall(\omega, \tau) \in B_{r} \cap\left[\omega \geqslant \omega_{0}\right], \\
\tilde{p}(\omega, \tau)>0 & \forall(\omega, \tau) \in B_{r} \cap\left[\omega<\omega_{0}\right] .\end{cases}
\end{aligned}
$$

Proof. (i) Since $u>y$ a.e. in $\mathcal{T}_{h}\left(B_{r}\right)$, we have $g=0$ a.e. in $\mathcal{T}_{h}\left(B_{r}\right)$ and then by (1.4), $u$ is $\mathcal{A}$-harmonic in this domain. It follows by Lemma 3.1 that $u>y$ or $u=y$ in $\mathcal{T}_{h}\left(B_{r}\right)$, which contradicts (i).

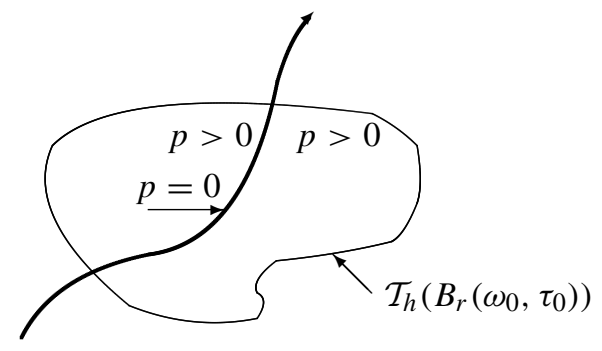

FIG. 6

(ii) Let $\xi \in \mathcal{D}\left(\mathcal{T}_{h}\left(B_{r}\right)\right), \xi \geqslant 0$. Using the fact that $\pm \xi$ are test functions for (P) and applying the changes of variables $T_{h}$ and $S_{h}$, we obtain, as in the proof of Theorem 2.1,

$$
\int_{\mathcal{T}_{h}\left(B_{r}\right)} \mathcal{A}(X, \nabla u) \cdot \nabla \xi \mathrm{d} X=\int_{\mathcal{T}_{h}\left(B_{r}\right)} g \mathcal{A}(X, e) \cdot \nabla \xi \mathrm{d} X=\int_{B_{r}} \tilde{g} \cdot \mathcal{Y}_{h} \cdot \frac{\partial \tilde{\xi}}{\partial \tau} \mathrm{d} \omega \mathrm{d} \tau .
$$

Now by Lemma 4.4, we have $\widetilde{g}=1$ a.e. in $B_{r} \cap\left[\omega<\omega_{0}\right]$ and then

$$
\begin{aligned}
\int_{T_{h} \circ S_{h}^{-1}\left(B_{r}\right)} \mathcal{A}(X, \nabla u) \cdot \nabla \xi \mathrm{d} X=\int_{B_{r} \cap\left[\omega<\omega_{0}\right]} \mathcal{Y}_{h} \cdot \frac{\partial \widetilde{\xi}}{\partial \tau} \mathrm{d} \omega \mathrm{d} \tau \\
=\int_{B_{r} \cap\left[\omega<\omega_{0}\right]}-\widetilde{\xi} \frac{\partial \mathcal{Y}_{h}}{\partial \tau} \mathrm{d} \omega \mathrm{d} \tau+\int_{S} \mathcal{Y}_{h} \widetilde{\xi} \nu_{\tau}=\int_{B_{r} \cap\left[\omega<\omega_{0}\right]}-\widetilde{\xi} \frac{\partial \mathcal{Y}_{h}}{\partial \tau} \mathrm{d} \omega \mathrm{d} \tau .
\end{aligned}
$$

FIG. 7 
It follows that

$$
\begin{aligned}
\int_{\mathcal{T}_{h}\left(B_{r}\right)}(\mathcal{A}(X, \nabla u)-\mathcal{A}(X, \nabla y)) \cdot \nabla \xi \mathrm{d} X & =\int_{B_{r}} \widetilde{\xi} \frac{\partial \mathcal{Y}_{h}}{\partial \tau} \mathrm{d} \omega \mathrm{d} \tau-\int_{B_{r} \cap\left[\omega<\omega_{0}\right]} \tilde{\xi} \frac{\partial \mathcal{Y}_{h}}{\partial \tau} \mathrm{d} \omega \mathrm{d} \tau \\
& =\int_{B_{r} \cap\left[\omega>\omega_{0}\right]} \widetilde{\xi} \frac{\partial \mathcal{Y}_{h}}{\partial \tau} \mathrm{d} \omega \mathrm{d} \tau \geqslant 0 .
\end{aligned}
$$

Since $u \geqslant y$ in $\mathcal{T}_{h}\left(B_{r}\right), u=y$ in $\mathcal{T}_{h}\left(B_{r} \cap\left[\omega \leqslant \omega_{0}\right]\right), \nabla y=e \neq 0$, we deduce from Lemma 3.1 that $u=y$ in $\mathcal{T}_{h}\left(B_{r}\right)$, which contradicts the fact that $u>y$ in $\mathcal{T}_{h}\left(B_{r} \cap\left[\omega>\omega_{0}\right]\right)$.

(iii) Argue as in (ii).

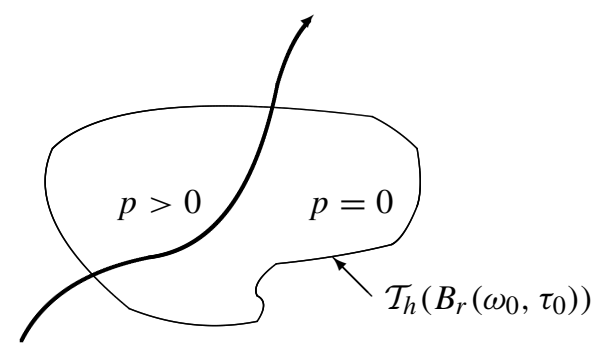

FIG. 8

\section{Continuity of the free boundary}

In this section we assume that $\mathcal{A}$ is strictly monotone in the following sense:

$$
(\mathcal{A}(X, \xi)-\mathcal{A}(X, \zeta)) \cdot(\xi-\zeta)>0 \quad \forall \xi \neq \zeta, \forall X \in \Omega .
$$

Then we have

THEOREM 5.1 For each $h \in \pi_{y}(\Omega)$, the function $\Phi_{h}$ defined in (3.4) is continuous on $\pi_{x}(\Omega \cap$ $[y=h])$.

Proof. It suffices to prove that $\Phi_{h}$ is upper semicontinuous. Let $X_{0}=\mathcal{T}_{h}\left(\omega_{0}, \tau_{0}\right) \in \Omega \cap \partial[p>0]$ and let $\epsilon>0$ be small enough. Thanks to Theorem 3.1 and Remark 3.2 we have necessarily $X\left(\alpha_{+}\left(\omega_{0}\right), \omega_{0}\right) \notin S_{3}$. Two cases are to be distinguished:

(i) First we assume that $X\left(\alpha_{+}\left(\omega_{0}\right), \omega_{0}\right) \notin \bar{S}_{3}$, where $\bar{S}_{3}$ denotes the closure of $S_{3}$ relative to $\partial \Omega$. Since $p\left(X_{0}\right)=\tilde{p}\left(\omega_{0}, \tau_{0}\right)=0$ and $p, \alpha_{+}$are continuous, there exists a ball $B_{\epsilon^{\prime}}\left(\omega_{0}, \tau_{0}\right)\left(0<\epsilon^{\prime}<\epsilon\right)$ such that

$$
\left\{\begin{array}{l}
\widetilde{p}(\omega, \tau) \leqslant \epsilon \quad \forall(\omega, \tau) \in B_{\epsilon^{\prime}}\left(\omega_{0}, \tau_{0}\right), \\
X\left(\alpha_{+}(\omega), \omega\right) \notin \bar{S}_{3} \quad \forall \omega \in\left(\omega_{0}-\epsilon^{\prime}, \omega_{0}+\epsilon^{\prime}\right) .
\end{array}\right.
$$

By Lemma 4.5, one of the following situations occurs:

$$
\begin{array}{llll}
\text { (a) } \exists\left(\omega_{1}, \tau_{1}\right) \in B_{\epsilon^{\prime}}\left(\omega_{0}, \tau_{0}\right) & \text { such that } \omega_{1}<\omega_{0}, & \tilde{p}\left(\omega_{1}, \tau_{1}\right)=0, \\
\text { (b) } \exists\left(\omega_{2}, \tau_{2}\right) \in B_{\epsilon^{\prime}}\left(\omega_{0}, \tau_{0}\right) & \text { such that } \omega_{2}>\omega_{0}, & \tilde{p}\left(\omega_{2}, \tau_{2}\right)=0 .
\end{array}
$$

Assume that for example (a) holds and set $X_{1}=\mathcal{T}_{h}\left(\omega_{1}, \tau_{1}\right)$ and $\tau_{M}=\max \left(\tau_{0}, \tau_{1}\right)$ (see Figure 9). 


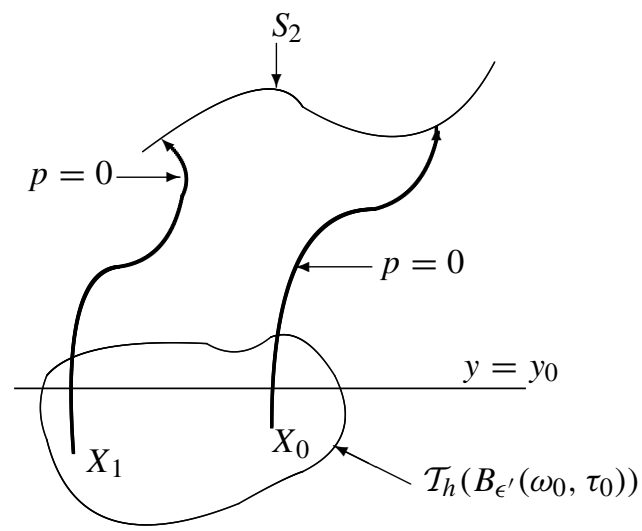

FIG. 9

Then by Theorem 3.1 we have

$$
\widetilde{p}\left(\omega_{i}, \tau\right)=0 \quad \forall\left(\omega_{i}, \tau\right) \in S_{h}\left(D_{h}\right) \text { such that } \tau>\tau_{M}(i=0,1) .
$$

Set $Z_{\tau_{M}}=\mathcal{T}_{h}\left(\left(\omega_{1}, \omega_{0}\right) \times\left(\tau_{M},+\infty\right) \cap S_{h}\left(D_{h}\right)\right)$ and let $y_{0} \in \mathbb{R}$ be such that $\mathcal{T}_{h}^{-1}\left(\left[y=y_{0}\right]\right) \cap$ $B_{\epsilon^{\prime}}\left(\omega_{0}, \tau_{0}\right) \cap\left[\tau>\tau_{M}\right] \neq \emptyset$. Note that

$$
\begin{aligned}
\mathcal{T}_{h}^{-1}\left(\left[y=y_{0}\right]\right) & \\
& =\left\{\left(\omega, \tau_{y_{0}}(\omega)\right) \in S_{h}\left(D_{h}\right)\left|\tau_{y_{0}}(\omega)=\int_{\alpha_{-}(\omega)}^{t_{y_{0}}(\omega)}\right| \mathcal{A}(X(s, \omega), e) \mid \mathrm{d} s, X_{2}\left(t_{y_{0}}(\omega), \omega\right)=y_{0}\right\} .
\end{aligned}
$$

Set

$$
\left\{\begin{array}{l}
D_{y_{0}, \tau_{M}}=\left[y>y_{0}\right] \cap Z_{\tau_{M}} \neq \emptyset, \\
v(y)=\left(\epsilon+y_{0}-y\right)^{+}+y, \\
\xi(x, y)=\chi\left(D_{y_{0}, \tau_{M}}\right)(u-v)^{+} .
\end{array}\right.
$$

Since $v \geqslant y=u$ on $\partial D_{y_{0}, \tau_{M}} \backslash\left(\left[y=y_{0}\right]\right)$, we have $\xi=0$ on $\partial D_{y_{0}, \tau_{M}} \backslash\left(\left[y=y_{0}\right]\right)$. Moreover $v\left(y_{0}\right)=\epsilon+y_{0} \geqslant u\left(x, y_{0}\right)$ and then $\xi\left(x, y_{0}\right)=0$. It follows that $\xi=0$ on $\partial D_{y_{0}, \tau_{M}}$. So $\pm \xi$ are test functions for $(\mathrm{P})$ and we have

$$
\int_{D_{y_{0}, \tau_{M}}}(\mathcal{A}(X, \nabla u)-g \mathcal{A}(X, e)) \cdot \nabla(u-v)^{+} \mathrm{d} X=0 .
$$

We also have

$$
\int_{D_{y_{0}, \tau_{M}}}(\mathcal{A}(X, \nabla v)-\chi([v=y]) \mathcal{A}(X, e)) \cdot \nabla(u-v)^{+} \mathrm{d} X=0 .
$$

Subtracting (5.3) from (5.2), we obtain

$$
\begin{aligned}
\left.\int_{D_{y_{0}, \tau_{M} \cap[v>y]}(\mathcal{A}(X, \nabla u)}-\mathcal{A}(X, \nabla v)\right) \cdot \nabla(u-v)^{+} \mathrm{d} X \\
+\int_{D_{y_{0}, \tau_{M} \cap[v=y]}(\mathcal{A}(X, \nabla u)-g \mathcal{A}(X, e)) \cdot \nabla(u-y) \mathrm{d} X=0 .}
\end{aligned}
$$


By Lemma 4.1, for $\left[y>y_{0}+\epsilon\right] \cap Z_{\tau_{M}}=D_{y_{0}, \tau_{M}} \cap[v=y]$ we have

$$
\int_{D_{y_{0}, \tau_{M} \cap[v=y]}}(\mathcal{A}(X, \nabla u)-g \mathcal{A}(X, e)) \cdot e \mathrm{~d} X \leqslant 0 .
$$

Adding (5.4) and (5.5), by taking into account (P)(i) we get

$$
\begin{aligned}
& \int_{D_{y_{0}, \tau_{M} \cap[v>y]}}(\mathcal{A}(X, \nabla u)-\mathcal{A}(X, \nabla v)) \cdot \nabla(u-v)^{+} \mathrm{d} X \\
& +\int_{D_{y_{0}, \tau_{M}} \cap[v=y] \cap[u>y]} \mathcal{A}(X, \nabla u) \cdot \nabla u \mathrm{~d} X+\int_{D_{y_{0}, \tau_{M}} \cap[v=y] \cap[u=y]}(1-g) \mathcal{A}(X, e) \cdot e \mathrm{~d} X \leqslant 0 .
\end{aligned}
$$

Since the three integrals on the left hand side of the above inequality are all nonnegative, we conclude by (5.1) that $\nabla(u-v)^{+}=0$ a.e. in $D_{y_{0}, \tau_{M}} \cap[v>y]$ and then, since $(u-v)^{+}=0$ on $\partial D_{y_{0}, \tau_{M}}$, we get $u \leqslant v$ in $D_{y_{0}, \tau_{M}} \cap[v>y]$. This leads to $p\left(x, y_{0}+\epsilon\right)=0$ for all $x \in \pi_{x}\left(D_{y_{0}, \tau_{M}}\right)$. Now for each $\omega \in\left(\omega_{1}, \omega_{0}\right)$, there exists a unique $t_{y_{0}, \epsilon}(\omega) \in\left(\alpha_{-}(\omega), \alpha_{+}(\omega)\right)$ such that

$$
X_{2}\left(t_{y_{0}, \epsilon}(\omega), \omega\right)=y_{0}+\epsilon, \quad p\left(X_{1}\left(t_{y_{0}, \epsilon}(\omega), \omega\right), y_{0}+\epsilon\right)=0,
$$

and if $\tau_{y_{0}, \epsilon}(\omega)=L_{y_{0}+\epsilon}\left(t_{y_{0}, \epsilon}(\omega), \omega\right)$, then we obtain $\tilde{p}\left(\omega, \tau_{y_{0}, \epsilon}(\omega)\right)=p \circ T_{h}\left(t_{y_{0}, \epsilon}(\omega), \omega\right)=$ $p\left(X_{1}\left(t_{y_{0}, \epsilon}(\omega), \omega\right), y_{0}+\epsilon\right)=0$ for all $\omega \in\left(\omega_{1}, \omega_{0}\right)$. Therefore $\Phi_{h}(\omega) \leqslant \tau_{y_{0}, \epsilon}(\omega)$. But since $X_{2}$ is increasing with respect to $t$, and $X_{2}\left(t_{y_{0}}(\omega), \omega\right)=y_{0}$ and $X_{2}\left(t_{y_{0}, \epsilon}(\omega), \omega\right)=y_{0}+\epsilon$, it follows that $t_{y_{0}}(\omega)<t_{y_{0}, \epsilon}(\omega)$ and then

$$
\begin{aligned}
\epsilon & =X_{2}\left(t_{y_{0}, \epsilon}(\omega), \omega\right)-X_{2}\left(t_{y_{0}}(\omega), \omega\right)=\int_{t_{y_{0}}(\omega)}^{t_{y_{0}, \epsilon}(\omega)} a^{2}(X(s, \omega)) \mathrm{d} s \\
& \geqslant \lambda\left(t_{y_{0}, \epsilon}(\omega)-t_{y_{0}}(\omega)\right)
\end{aligned}
$$

and

$$
\begin{aligned}
\tau_{y_{0}, \epsilon}(\omega) & =\tau_{y_{0}}(\omega)+\int_{t_{y_{0}}(\omega)}^{t_{y_{0}, \epsilon}(\omega)}|\mathcal{A}(X(s, \omega), e)| \mathrm{d} s \\
& \leqslant \tau_{y_{0}}(\omega)+M\left(t_{y_{0}, \epsilon}(\omega)-t_{y_{0}}(\omega)\right) \leqslant \tau_{y_{0}}(\omega)+\frac{M}{\lambda} \epsilon .
\end{aligned}
$$

So

$$
\Phi_{h}(\omega) \leqslant \tau_{y_{0}}(\omega)+\frac{M}{\lambda} \epsilon<\tau_{0}+\epsilon^{\prime}+\frac{M}{\lambda} \epsilon<\Phi_{h}\left(\omega_{0}\right)+\left(1+\frac{M}{\lambda}\right) \epsilon \quad \forall \omega \in\left(\omega_{1}, \omega_{0}\right)
$$

Hence $\Phi_{h}$ is u.s.c. at $\omega_{0}$ from the left. Using Lemma 4.5 and arguing as above, one can prove that $\Phi_{h}$ is u.s.c. at $\omega_{0}$ from the right. Thus $\Phi_{h}$ is continuous at $\omega_{0}$.

(ii) Now we assume that $X\left(\alpha_{+}\left(\omega_{0}\right), \omega_{0}\right) \in \bar{S}_{3} \backslash S_{3}$. Then one of the following situations holds:
(a) $\exists \eta>0 \forall \omega \in\left(\omega_{0}-\eta, \omega_{0}+\eta\right) \quad X\left(\alpha_{+}(\omega), \omega\right) \in S_{3} \Leftrightarrow \omega \in\left(\omega_{0}, \omega_{0}+\eta\right)$
(b) $\exists \eta>0 \forall \omega \in\left(\omega_{0}-\eta, \omega_{0}+\eta\right) \quad X\left(\alpha_{+}(\omega), \omega\right) \in S_{3} \Leftrightarrow \omega \in\left(\omega_{0}-\eta, \omega_{0}\right)$. 
Assume for example that (a) holds. Then it is easy to see that

$$
\Phi_{h}(\omega)=L_{h}\left(\alpha_{+}(\omega), \omega\right) \quad \forall \omega \in\left(\omega_{0}, \omega_{0}+\eta\right) .
$$

Arguing as in (i) one can prove that $\Phi_{h}$ is continuous at $\omega_{0}$ from the left. On the other hand, we deduce from (5.6) that $u>y$ in a right neighborhood of the curve $X\left(\cdot, \omega_{0}\right)$. Using the continuity from the left and Lemma 4.5, we have necessarily $\Phi_{h}\left(\omega_{0}\right)=L_{h}\left(\alpha_{+}\left(\omega_{0}\right), \omega_{0}\right)$. Therefore we now have

$$
\Phi_{h}(\omega)=L_{h}\left(\alpha_{+}(\omega), \omega\right) \quad \forall \omega \in\left[\omega_{0}, \omega_{0}+\eta\right)
$$

which leads to the continuity of $\Phi_{h}$ from the right at $\omega_{0}$.

We argue similarly if (b) holds.

REMARK $5.1 \quad$ (i) For each $X_{0} \in \Omega \cap \partial[p>0]$, there exists $h \in \pi_{y}(\Omega)$ such that $X_{0} \in$ $T_{h}\left(D_{h}\right) \cap \partial[p>0]$ and then $X_{0}=\mathcal{T}_{h}\left(\omega_{0}, \tau_{0}\right)$ with $\tau_{0}=\Phi_{h}\left(\omega_{0}\right)$. Therefore from Theorem 5.1 , the free boundary is represented in a neighborhood of $X_{0}$ by the graph of the continuous function $\Phi_{h}$.

(ii) Since the free boundary is now represented locally by graphs of continuous functions, it follows in particular that $\partial[p>0]$ is of Lebesgue measure zero.

The following result expresses the fact that $g$ is the characteristic function of the dry region.

COROLlary 5.1 Let $(u, g)$ be a solution of $(\mathrm{P})$. Then

$$
g=\chi([p=0])=\chi([u=y]) .
$$

Proof. First by (P)(i), we have $g=0$ in $[p>0]$. Next if $\left(x_{0}, y_{0}\right) \in \Omega \backslash \overline{[p>0]}$, then since $\Omega \backslash \overline{[p>0]}$ is an open set there exists $\epsilon_{0}>0$ small enough and $h \in \pi_{y}(\Omega)$ such that $B_{\epsilon_{0}}\left(x_{0}, y_{0}\right) \subset$ $(\Omega \backslash \overline{[p>0]}) \cap T_{h}\left(D_{h}\right)$.

By Lemma $4.4, \widetilde{g}=1$ a.e. in $\mathcal{T}_{h}^{-1}\left(B_{\epsilon_{0}}\left(x_{0}, y_{0}\right)\right)$ or equivalently $g=1$ in $B_{\epsilon_{0}}\left(x_{0}, y_{0}\right)$. Therefore $g=1$ in $\Omega \backslash \overline{[p>0]}$.

Since the set $\partial[p>0] \cap \Omega$ is of Lebesgue measure zero, we conclude that $g=\chi([p=0])=$ $\chi([u=y])$.

\section{Uniqueness of reservoirs-connected solutions}

Definition 6.1 A solution $(u, g)$ of $(\mathrm{P})$ is a reservoirs-connected solution if for each connected component $C$ of $[u>y]$, we have $\bar{C} \cap S_{3} \neq \emptyset$.

REMARK 6.1 Thanks to Remark 3.2, if $\bar{C} \supset S_{3, i}(i=1, \ldots, N)$, then $C$ contains the strip of $\Omega$ below $S_{3, i}$ in the following sense:

$$
\bigcup_{h \in \pi_{y}(\Omega)}\left(\mathcal{T}_{h}\left(\left(\pi_{\omega}\left(\mathcal{S}_{3, i}^{h}\right) \times \mathbb{R}\right) \cap S_{h}\left(D_{h}\right)\right) \subset C, \quad \text { where } \quad \mathcal{S}_{3, i}^{h}=\mathcal{T}_{h}^{-1}\left(S_{3, i}\right) .\right.
$$

THEOREM 6.1 Let $(u, g)$ be a solution of $(\mathrm{P})$ and $C$ a connected component of $[u>y]$ such that $\bar{C} \cap S_{3}=\emptyset$. Set $h_{C}=\sup \{y \mid(x, y) \in C\}$. Then

$$
u(x, y)=\left(h_{C}-y\right)^{+} \chi(C)+y, \quad g=1-\chi(C)
$$


for

$$
(x, y) \in \bigcup_{h \in \pi_{y}(\Omega)}\left(\mathcal{T}_{h}\left(\pi_{\omega}\left(\mathcal{C}_{h}\right) \times \mathbb{R} \cap S_{h}\left(D_{h}\right)\right)\right), \quad \text { where } \quad \mathcal{C}_{h}=\mathcal{T}_{h}^{-1}\left(C \cap T_{h}(\Omega)\right) .
$$

Proof. Since $\pm \chi(C)(u-y)$ are test functions for $(\mathrm{P})$, we have

$$
\int_{C}(\mathcal{A}(X, \nabla u)-g \mathcal{A}(X, e)) \cdot \nabla(u-y) \mathrm{d} X=0 .
$$

By Lemma 4.3, we have

$$
\int_{C}(\mathcal{A}(X, \nabla u)-g \mathcal{A}(X, e)) \cdot e \mathrm{~d} X \leqslant 0
$$

Adding (6.1) and (6.2), we obtain

$$
\int_{C \cap[u>y]} \mathcal{A}(X, \nabla u) \cdot \nabla u \mathrm{~d} X+\int_{C \cap[u=y]}(1-g) \mathcal{A}(X, e) \cdot e \mathrm{~d} X \leqslant 0 .
$$

It follows that $\nabla u=0$ a.e. in $C$ and so $u$ is equal to some positive constant $k$ in $C$, which can be easily verified to be equal to $h_{C}$.

Using Theorem 3.1 and (5.7) we deduce that $u=\left(h_{C}-y\right)^{+} \chi(C)+y$ and $g=1-\chi(C)$ in $\bigcup_{h \in \pi_{y}(\Omega)}\left(\mathcal{T}_{h}\left(\pi_{\omega}\left(\mathcal{C}_{h}\right) \times \mathbb{R} \cap S_{h}\left(D_{h}\right)\right)\right)$.

DeFINITION 6.2 We define a pool in $\Omega$ to be a pair $(\bar{u}, \bar{g})$ of functions defined in $\Omega$ by

$$
\bar{u}=\left(h_{C}-y\right)^{+} \chi(C)+y \quad \text { and } \quad \bar{g}=1-\chi(C) \text { a.e. in } \Omega,
$$

where $C$ is a subdomain of $\Omega$ and $h_{C}=\max \{y \mid(x, y) \in C\}$.

THEOREM 6.2 Each solution of $(\mathrm{P})$ can be written as the sum of a reservoirs-connected solution and pools.

Proof. See [14], [15], [17] and [27].

In order to prove the uniqueness of the reservoirs-connected solution we assume that $S_{3}$ is of class $C^{1, \alpha}$, and $\Omega$ is covered by a finite number of sets $T_{h}\left(D_{h}\right)$, that is, there are $h_{1}, \ldots, h_{n} \in \pi_{y}(\Omega)$ such that

$$
\Omega=\bigcup_{k=1}^{n} T_{h_{k}}\left(D_{h_{k}}\right)
$$

We also assume that

$$
\mathcal{A}(X, \xi)=|a(X) \xi \cdot \xi|^{(q-2) / 2} a(X) \xi, \quad \text { where } \quad a(X) \in C^{1}(\bar{\Omega}) \text { is a 2-by-2 matrix. }
$$

Then we can state our uniqueness theorem

THEOREM 6.3 Under assumptions (6.3)-(6.4), the reservoirs-connected solution is unique.

The proof of Theorem 6.3 requires three lemmas. 
LEMmA 6.1 Let $\left(u_{1}, g_{1}\right)$ and $\left(u_{2}, g_{2}\right)$ be two solutions of $(\mathrm{P})$. Then for $i=1,2$ and $\zeta \in W^{1, q}(\Omega)$ we have

$$
\mathcal{F}_{i}(\zeta)=\int_{\Omega}\left(\left(\mathcal{A}\left(X, \nabla u_{i}\right)-\mathcal{A}\left(X, \nabla u_{m}\right)\right)-\left(g_{i}-g_{M}\right) \mathcal{A}(X, e)\right) \cdot \nabla \zeta \mathrm{d} X=0,
$$

where $u_{m}=\min \left(u_{1}, u_{2}\right)$ and $g_{M}=\max \left(g_{1}, g_{2}\right)$.

To prove Lemma 6.1 we need another lemma:

LEMma 6.2 Let $\left(u_{1}, g_{1}\right)$ and $\left(u_{2}, g_{2}\right)$ be two solutions of $(\mathrm{P})$ and $\Phi_{h_{k}}^{i}(i=1,2$ and $k=1, \ldots, n)$ be the corresponding free boundary functions. Then for $i=1,2$ and $\zeta \in W^{1, q}(\Omega) \cap C^{0}(\bar{\Omega})$ with $\zeta \geqslant 0$ we have

$$
\mathcal{F}_{i}(\zeta) \leqslant \sum_{k=1}^{n} \int_{\mathcal{D}_{h_{k}}^{i}} \mathcal{Y}_{h_{k}}\left(\omega, \Phi_{h_{k}}^{i}(\omega)\right) \cdot \zeta \circ T_{h_{k}} \circ S_{h_{k}}^{-1}\left(\omega, \Phi_{h_{k}}^{i}(\omega)\right) \mathrm{d} \omega,
$$

where $\mathcal{D}_{h_{k}}^{i}=\left\{\omega \in \pi_{\omega}\left(S_{h_{k}}\left(D_{h_{k}}\right)\right) \mid \Phi_{h_{k}}^{0}(\omega)=\min \left(\Phi_{h_{k}}^{1}(\omega), \Phi_{h_{k}}^{2}(\omega)\right)<\Phi_{h_{k}}^{i}(\omega)\right\}$.

Proof. First thanks to (6.3) there exists a partition of unity $\left(\theta_{k}\right)_{k=1}^{n}$ corresponding to the open covering $\left(T_{h_{k}}\left(D_{h_{k}}\right)\right)_{k=1}^{n}$ of $\Omega$, that is,

$$
\theta_{k} \in \mathcal{D}\left(T_{h_{k}}\left(D_{h_{k}}\right)\right), \quad 0 \leqslant \theta_{k} \leqslant 1 \quad \forall k=1, \ldots, n, \quad \sum_{k=1}^{n} \theta_{k}=1 \quad \text { in } \Omega .
$$

Let $\zeta \in W^{1, q}(\Omega) \cap C^{0}(\bar{\Omega}), \zeta \geqslant 0$ and let $\zeta_{k}=\theta_{k} \zeta$. Since $\mathcal{F}_{i}(\zeta)=\sum_{k=1}^{n} \mathcal{F}_{i}\left(\zeta_{k}\right)$, it suffices to show that

$$
\mathcal{F}_{i}\left(\zeta_{k}\right) \leqslant \int_{\mathcal{D}_{h_{k}}^{i}} \mathcal{Y}_{h_{k}}\left(\omega, \Phi_{h_{k}}^{i}(\omega)\right) \cdot \zeta_{k} \circ T_{h_{k}} \circ S_{h_{k}}^{-1}\left(\omega, \Phi_{h_{k}}^{i}(\omega)\right) \mathrm{d} \omega .
$$

So let $\epsilon>0$ and $\xi_{k}=\min \left(\zeta_{k},\left(u_{1}-u_{2}\right)^{+} / \epsilon\right)$. Clearly $\pm \xi_{k}$ are test functions for (P) and we have

$$
\int_{T_{h_{k}}\left(D_{h_{k}}\right)}\left(\left(\mathcal{A}\left(X, \nabla u_{1}\right)-\mathcal{A}\left(X, \nabla u_{2}\right)\right)-\left(g_{1}-g_{2}\right) \mathcal{A}(X, e)\right) \cdot \nabla \xi_{k} \mathrm{~d} X=0 .
$$

Since we integrate only on the set $\left[u_{1}>u_{2}\right]$ where $u_{2}=u_{m}$, (6.7) is equivalent to $\mathcal{F}_{1}\left(\xi_{k}\right)=0$, which can be written as

$$
\begin{aligned}
& \int_{T_{h_{k}}\left(D_{h_{k}}\right) \cap\left[\left(u_{1}-u_{2}\right)^{+}>\epsilon \zeta_{k}\right]}\left(\left(\mathcal{A}\left(X, \nabla u_{1}\right)-\mathcal{A}\left(X, \nabla u_{m}\right)\right) \cdot \nabla \zeta_{k} \mathrm{~d} X-\int_{T_{h_{k}\left(D_{h_{k}}\right)}}\left(g_{1}-g_{M}\right) \mathcal{A}(X, e) \cdot \nabla \zeta_{k} \mathrm{~d} X\right. \\
& \leqslant-\int_{T_{h_{k}}\left(D_{h_{k}}\right)}\left(g_{1}-g_{M}\right) \mathcal{A}(X, e) \cdot \nabla\left(\zeta_{k}-\frac{\left(u_{1}-u_{2}\right)^{+}}{\epsilon}\right)^{+} \mathrm{d} X=I_{\epsilon}^{k} .
\end{aligned}
$$

Using the $C^{1}$ diffeomorphisms $T_{h_{k}}$ and $S_{h_{k}}$, for $\tilde{g}_{1 k}=g_{1} \circ T_{h_{k}} \circ S_{h_{k}}^{-1}$ and $\tilde{g}_{M k}=g_{M} \circ T_{h_{k}} \circ S_{h_{k}}^{-1}$ we obtain

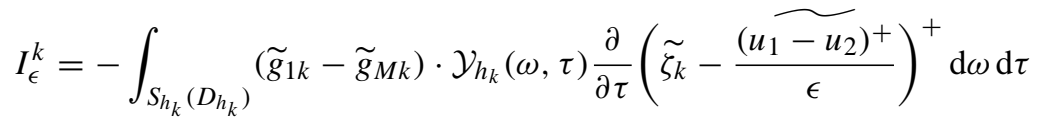




$$
\begin{aligned}
& =\int_{\left[\tilde{p}_{1 k}>0=\tilde{p}_{m k}=\tilde{p}_{2 k}\right]} \mathcal{Y}_{h_{k}}(\omega, \tau) \frac{\partial}{\partial \tau}\left(\widetilde{\zeta_{k}}-\frac{\left.\widetilde{\left(u_{1}-u_{2}\right.}\right)^{+}}{\epsilon}\right)^{+} \mathrm{d} \omega \mathrm{d} \tau \\
& =\int_{\left[\Phi_{h_{k}}^{2}(\omega)<\tau<\Phi_{h_{k}}^{1}(\omega)\right]} \mathcal{Y}_{h_{k}}(\omega, \tau) \frac{\partial}{\partial \tau}\left(\widetilde{\widetilde{\zeta}_{k}}-\frac{\left(\widetilde{u_{1}-u_{2}}\right)^{+}}{\epsilon}\right)^{+} \mathrm{d} \omega \mathrm{d} \tau \\
& =\int_{\mathcal{D}_{h_{k}}^{1}} \int_{\Phi_{h_{k}}^{2}(\omega)}^{\Phi_{h_{k}}^{1}(\omega)} \mathcal{Y}_{h_{k}}(\omega, \tau) \frac{\partial}{\partial \tau}\left(\widetilde{\zeta_{k}}-\frac{\left(\widetilde{u_{1}-u_{2}}\right)^{+}}{\epsilon}\right)^{+} \mathrm{d} \omega \mathrm{d} \tau .
\end{aligned}
$$

By the second mean value theorem there exists $\Phi_{k}^{*}(\omega) \in\left[\Phi_{h_{k}}^{2}(\omega), \Phi_{h_{k}}^{1}(\omega)\right]$ such that

$$
\begin{aligned}
I_{\epsilon}^{k} & =\int_{\mathcal{D}_{h_{k}}^{1}} \mathcal{Y}_{h_{k}}\left(\omega, \Phi_{k}^{1}(\omega)\right) \int_{\Phi_{k}^{*}(\omega)}^{\Phi_{h_{k}}^{1}(\omega)} \frac{\partial}{\partial \tau}\left(\widetilde{\zeta_{k}}-\frac{\left(\widetilde{u_{1}-u_{2}}\right)^{+}}{\epsilon}\right)^{+} \mathrm{d} \omega \mathrm{d} \tau \\
& \leqslant \int_{\mathcal{D}_{h_{k}}^{1}} \mathcal{Y}_{h_{k}}\left(\omega, \Phi_{h_{k}}^{1}(\omega)\right) \cdot \widetilde{\zeta_{k}}\left(\omega, \Phi_{h_{k}}^{1}(\omega)\right) \mathrm{d} \omega .
\end{aligned}
$$

It follows that

$$
\begin{aligned}
& \int_{T_{h_{k}}\left(D_{h_{k}}\right) \cap\left[\left(u_{1}-u_{2}\right)^{+}>\epsilon \zeta_{k}\right]}\left(\mathcal{A}\left(X, \nabla u_{1}\right)-\mathcal{A}\left(X, \nabla u_{m}\right)\right) \cdot \nabla \zeta_{k} \mathrm{~d} X \\
& \quad-\int_{T_{h_{k}}\left(D_{h_{k}}\right)}\left(g_{1}-g_{M}\right) \mathcal{A}(X, e) \cdot \nabla \zeta_{k} \mathrm{~d} X \leqslant \int_{\mathcal{D}_{h_{k}}^{1}} \mathcal{Y}_{h_{k}}\left(\omega, \Phi_{h_{k}}^{1}(\omega)\right) \cdot \widetilde{\zeta_{k}}\left(\omega, \Phi_{h_{k}}^{1}(\omega)\right) \mathrm{d} \omega .
\end{aligned}
$$

Letting $\epsilon$ go to 0 , we get (6.6) for $i=1$. The proof for $i=2$ is similar.

Proof of Lemma 6.1. Let $\zeta \in C^{1}(\bar{\Omega}), \zeta \geqslant 0$. For $\delta>0$ we set

$$
A_{m}=\bigcup_{k=1}^{n}\left[\tilde{p}_{m k}>0\right], \quad \alpha_{\delta}(\omega, \tau)=\left(1-\frac{d\left((\omega, \tau), A_{m}\right)}{\delta}\right)^{+}, \quad h_{\delta}=\alpha_{\delta} \circ S_{h_{k}} \circ T_{h_{k}}^{-1}
$$

and remark that $\mathcal{F}_{1}(\zeta)=\mathcal{F}_{1}\left(h_{\delta} \zeta\right)+\mathcal{F}_{1}\left(\left(1-h_{\delta}\right) \zeta\right)$. By the previous lemma we have

$$
\mathcal{F}_{1}\left(h_{\delta} \zeta\right) \leqslant \sum_{k=1}^{n} \int_{\mathcal{D}_{h_{k}}^{1}} \mathcal{Y}_{h_{k}}\left(\omega, \Phi_{h_{k}}^{1}(\omega)\right) \widetilde{\zeta}_{k}\left(\omega, \Phi_{h_{k}}^{1}(\omega)\right) \alpha_{\delta}\left(\omega, \Phi_{h_{k}}^{1}(\omega)\right) \mathrm{d} \omega
$$

Since $\left(1-h_{\delta}\right) \zeta$ is a test function for $(\mathrm{P})$, we have

$$
\int_{\Omega}\left(\mathcal{A}\left(X, \nabla u_{1}\right)-g_{1} \mathcal{A}(X, e)\right) \cdot \nabla\left(\left(1-h_{\delta}\right) \zeta\right) \mathrm{d} X \leqslant 0 .
$$

Moreover the function $1-\alpha_{\delta}$ vanishes on $\bar{A}_{m}$ and $g_{M}=1$ in $\Omega \backslash \bar{A}_{m}$. So

$$
\int_{\Omega}\left(\mathcal{A}\left(X, \nabla u_{m}\right)-g_{M} \mathcal{A}(X, e)\right) \cdot \nabla\left(\left(1-h_{\delta}\right) \zeta\right) \mathrm{d} X=0 .
$$


This leads to $\mathcal{F}_{1}\left(\left(1-h_{\delta}\right) \zeta\right) \leqslant 0$ and

$$
\mathcal{F}_{1}(\zeta) \leqslant \sum_{k=1}^{n} \int_{\mathcal{D}_{h_{k}}^{1}} \mathcal{Y}_{h_{k}}\left(\omega, \Phi_{h_{k}}^{1}(\omega)\right) \widetilde{\zeta}_{k}\left(\omega, \Phi_{h_{k}}^{1}(\omega)\right) \alpha_{\delta}\left(\omega, \Phi_{h_{k}}^{1}(\omega)\right) \mathrm{d} \omega
$$

Letting $\delta \rightarrow 0$, we deduce $\mathcal{F}_{1}(\zeta) \leqslant 0$. It is then easy (see [15], [17], [27]) to show that $\mathcal{F}_{1}(\zeta)=0$ for all $\zeta \in W^{1, q}(\Omega)$. Similarly we obtain $\mathcal{F}_{2}(\zeta)=0$ for all $\zeta \in W^{1, q}(\Omega)$.

LEMMA 6.3 Let $\Omega_{0}$ be a domain of $\mathbb{R}^{2}, \Gamma_{0} \subset \partial \Omega_{0}$ of class $C^{1, \alpha}$ and let $u_{1}, u_{2} \in W_{\text {loc }}^{1, q}\left(\Omega_{0}\right)$ be such that:

$$
\left\{\begin{aligned}
\text { (i) } \operatorname{div}\left(\mathcal{A}\left(X, \nabla u_{1}\right)\right)=\operatorname{div}\left(\mathcal{A}\left(X, \nabla u_{2}\right)\right)=0 \quad \text { in } \mathcal{D}^{\prime}\left(\Omega_{0}\right), \\
\text { (ii) } u_{1} \leqslant u_{2} \text { in } \Omega_{0}, \\
\text { (iii) } u_{1}=u_{2} \text { on } \Gamma_{0}, \quad u_{1}, u_{2} \in C^{1}\left(\Omega_{0} \cup \Gamma_{0}\right), \\
\text { (iv) } \mathcal{A}\left(X, \nabla u_{1}\right) \cdot v=\mathcal{A}\left(X, \nabla u_{2}\right) \cdot v \text { on } \Gamma_{0}, \\
\text { (v) } \nabla u_{1}(X) \neq 0 \quad \forall X \in \Gamma_{0} \text { or } \nabla u_{2}(X) \neq 0 \quad \forall X \in \Gamma_{0} .
\end{aligned}\right.
$$

Then $u_{1}=u_{2}$ in $\Omega$.

Proof. See [16].

Proof of Theorem 6.3. When $q=2$ the proof is given in [27]. For the general case we use Lemma 6.3.

Let $\left(u_{1}, g_{1}\right),\left(u_{2}, g_{2}\right)$ be two solutions of $(\mathrm{P})$. By Lemma 6.1 one can see that $\left(u_{m}, g_{M}\right)$ is also a solution of $(\mathrm{P})$. Let $C_{m, i}$ be the connected component of the set $\left[u_{m}>y\right]$ that contains $S_{3, i}$ on its boundary. By Lemma 6.1 we deduce easily that $u_{1}$ and $u_{m}$ satisfy the conditions (i) and (iv) of Lemma 6.3 with $\Omega_{0}=C_{m, i}$ and $\Gamma_{0}=S_{3, i}$.

The condition (ii) is obviously satisfied and the first part of (iii) is true since $u_{1}=u_{2}=\varphi+y$ on $S_{3}$. The second part of (iii) is also true (see [19] and [26]). So if we show that (v) is satisfied we will get $u_{1}=u_{m}$ in $C_{m, i}$. For this purpose, we distinguish two cases:

- If $\nabla u_{1}$ and $\nabla u_{m}$ are not identically zero on $\Gamma_{0}$ then by (iii) there exists $\Gamma_{0}^{\prime} \subset \Gamma_{0}$ such that $\nabla u_{1} \neq 0$ on $\Gamma_{0}^{\prime}$ or $\nabla u_{m} \neq 0$ on $\Gamma_{0}^{\prime}$. Therefore (v) is satisfied on $\Gamma_{0}^{\prime}$.

- If $\nabla u_{1}=0$ on $\Gamma_{0}$ and $\nabla u_{m}=0$ on $\Gamma_{0}$, then $u_{1}$ and $u_{m}$ are both constant along $\Gamma_{0}$. Since $u_{1}=u_{m}$ on $S_{3}$, it follows that $u_{1}=u_{m}=h_{i}$ on $\Gamma_{0}$ for some constant $h_{i}$. Therefore one can extend $u_{1}$ and $u_{m}$ into $B \backslash \Omega$ by $h_{i}$ where $B$ is a ball centered at a point of $S_{3, i}=\Gamma_{0}$ in such a way that $u_{j} \in C^{1}\left(C_{m, i} \cup B\right)$ and $\operatorname{div}\left(\mathcal{A}\left(X, \nabla u_{j}\right)\right)=0$ in $\mathcal{D}^{\prime}\left(C_{m, i} \cup B\right)$. So $\nabla u_{j}$ has nonisolated zeros and then (see [4]) $u_{j}=h_{i}$ in $C_{m, i}(j=1, m)$. Thus $u_{1}=u_{m}$ in $C_{m, i}$.

One can show as in [15], [17], [27] that $C_{1, i}=C_{m, i}$ and $u_{1}=u_{m}$ in $C_{1, i}$. In the same way we prove that $u_{2}=u_{m}$ in $C_{2, i}=C_{m, i}$. We conclude that $u_{1}=u_{2}$ in $C_{1, i}=C_{2, i}$ for all $i=1, \ldots, N$. This means that $u_{1}=u_{2}$ in $\left[u_{1}>y\right]=\left[u_{2}>y\right]$, which leads to $u_{1}=u_{2}$ in $\Omega$. Finally, we deduce from (5.7) that $g_{1}=g_{2}$ in $\Omega$.

REMARK 6.2 The assumption (6.4) is needed only to ensure the isolation of critical points of $\mathcal{A}$-harmonic functions (see [1]).

\section{Acknowledgments}

We would like to thank KFUPM for its support. The second author is grateful to Professor M. Chipot for fruitful discussions they had a few years ago. He also thanks Professor J. F. Rodrigues for some useful suggestions regarding the improvement of the final version of the paper. 


\section{REFERENCES}

1. Alessandrini, G. \& Sigalotti, M. Geometric properties of solutions to the anisotropic $p$-Laplace equation in dimension two. Ann. Acad. Sci. Fenn. Math. 26 (2001), 249-266. Zbl 1002.35044 MR 2002f:35081

2. Alt, H. W. A free boundary problem associated with the flow of ground water. Arch. Rat. Mech. Anal. 64 (1977), 111-126. Zbl 0371.76079 MR 55 \#1921

3. Alt, H. W. Strömungen durch inhomogene poröse Medien mit freiem Rand. J. Reine Angew. Math. 305 (1979), 89-115. Zbl 0392.76091 MR 80d:76086

4. Alt, H. W. The fluid flow through porous media. Regularity of the free surface. Manuscripta Math. 21 (1977), 255-272. Zbl 0371.76080 MR 56\#7475

5. Alt, H. W. \& Gilardi, G. The behavior of the free boundary for the dam problem. Ann. Scuola Norm. Sup. Pisa 9 (1981), 571-626. Zbl 0521.76092 MR 85c:35089a

6. BAiocchi, C. Su un problema di frontiera libera connesso a questioni di idraulica. Ann. Mat. Pura Appl. 92 (1972), 107-127. Zbl 0258.76069 MR 53\#12207

7. Baiocchi, C. \& CApelo, A. Variational and Quasivariational Inequalities. Applications to Free Boundary Problems. Wiley, New York (1984). Zbl 0551.49007 MR 86e:49018

8. Baiocchi, C., Comincioli, V., Magenes, E., \& Pozzi, G. A. Free boundary problems in the theory of fluid flow through porous media: existence and uniqueness theorems. Ann. Mat. Pura Appl. 97 (1973), 1-82. Zbl 0343.76036 MR 49 \#6772

9. BAIOcChi, C. \& Friedman, A. A filtration problem in a porous medium with variable permeability. Ann. Mat. Pura Appl. 114 (1977), 377-393. Zbl 0386.76080 MR 57 \#8362

10. Bear, J. \& Verruit, A. Modeling Groundwater Flow and Pollution. Reidel (1992).

11. Benci, V. On a filtration through a porous medium. Ann. Mat. Pura Appl. 100 (1974), 191-209. Zbl 0298.76049 MR 50 \#9144

12. Brézis, H., Kinderlehrer, D., \& Stampacchia, G. Sur une nouvelle formulation du problème de l'écoulement à travers une digue. C. R. Acad. Sci. Paris Sér. A 287 (1978), 711-714. Zbl 0391.76072 MR 80b:3513

13. Caffarelli, L. A. \& Friedman, A. The dam problem with two layers. Arch. Rat. Mech. Anal. 68 (1978), 125-154. Zbl 0397.76087 MR 81d:3508

14. Carrillo, J. \& Chipot, M. On the dam problem. J. Differential Equations 45 (1982), 234-271. Zbl 0504.35082

15. Carrillo, J. \& Lyaghfouri, A. The dam problem for nonlinear Darcy's laws and generalized boundary conditions. Ann. Scuola Norm. Sup. Pisa 26 (1998), 453-505. Zbl 0928.76111 MR 99f:35169

16. Challal, S. \& Lyaghfouri, A. On the behavior of the interface separating fresh and salt groundwater in a heterogeneous coastal aquifer. Electron. J. Differential Equations 2003, No. 44, 27 pp.

17. Chipot, M. \& Lyaghfouri, A. The dam problem with linear Darcy's law and leaky boundary conditions. Adv. Differential Equations 3 (1998), 1-50. Zbl 0947.35177| MR 99a:35276

18. Damascelli, L. Comparison theorems for some quasilinear degenerate elliptic operators and applications to symmetry and monotonicity results. Ann. Inst. H. Poincaré 15 (1998), 493-516. Zbl 0911.35009 MR 99e:35081

19. DiBenedetto, E. $C^{1}$ local regularity of weak solutions of degenerate elliptic equations. Nonlinear Anal. 7 (1983), 827-850. Zbl 0539.35027 MR 85d:3503

20. Dung, L. On a class of singular quasilinear elliptic equations with general structure and distribution data. Nonlinear Anal. 28 (1997), 1879-1902. Zbl 0874.35039 MR 98a:3503

21. Friedman, A. \& HuAng, S.-Y. The inhomogeneous dam problem with discontinuous permeability. Ann. Scuola Norm. Sup. Pisa 14 (1987), 49-77. Zbl 0644.35097 MR 90a:35233 
22. Greenkorn, R. A. Flow Phenomena in Porous Media: Fundamental and Applications in Petroleum, Water and Food Production. Dekker, New York (1983).

23. Hale, J. Ordinary Differential Equations. 2nd ed., Krieger, Huntington, NY (1980). Zbl 0433.34003 MR 82e:34001

24. KILPELÄINEN, T. Hölder continuity of solutions to quasilinear elliptic equations involving measures. Potential Anal. 3 (1994), 265-272. Zbl 0813.35016 |MR 95j:35071

25. LEWIS, J. L. Regularity of the derivatives of solutions to certain degenerate elliptic equations. Indiana Univ. Math. J. 32 (1983), 849-858. Zbl 0554.35048 MR 84m:35048

26. Lieberman, G. M. Boundary regularity for solutions of degenerate elliptic equations. Nonlinear Anal. 12 (1988), 1203-1219. Zbl 0675.35042

27. Lyaghfouri, A. The inhomogeneous dam problem with linear Darcy's law and Dirichlet boundary conditions. Math. Models Methods Appl. Sci. 8 (1996), 1051-1077. Zbl 0869.76088 MR 98a:35146

28. Lyaghfouri, A. On the uniqueness of the solution of a nonlinear filtration problem through a porous medium. Calc. Var. Partial Differential Equations 6 (1998), 67-94. Zbl 0909.35052 MR 98k:76135

29. Lyaghfouri, A. A unified formulation for the dam problem. Riv. Mat. Univ Parma 1 (1998), 113-148. Zbl 0929.76133 MR 2000c:35262

30. Rakotoson, J. M. Equivalence between the growth of $\int_{B(x, r)}|\nabla u|^{p} \mathrm{~d} y$ and $T$ in the equation $P[u]=T$. J. Differential Equations 86 (1990), 102-122. Zbl 0707.35033 MR 91k:35020

31. Rodrigues, J. F. Obstacle Problems in Mathematical Physics. North-Holland, Amsterdam (1987). Zbl 0606.73017 MR 88d:35006

32. StaVre, R. \& Vernescu, B. Incompressible fluid flow through a nonhomogeneous and anisotropic dam. Nonlinear Anal. 9 (1985), 799-810. Zbl 0535.35080 MR 86j:76062 